\title{
DEVELOPMENT OF A MEMS REPULSIVE \\ ACTUATOR FOR LARGE OUT-OF-PLANE
}

FORCE

by

\author{
Imran Khan
}

A thesis submitted in conformity with the requirements for the degree of Masters of Applied Science

Graduate Department of Mechanical and Industrial Engineering, University of Toronto

(C) Copyright by Imran Khan, 2013 


\title{
DEVELOPMENT OF A MEMS REPULSIVE ACTUATOR FOR LARGE OUT-OF-PLANE FORCE
}

\author{
Imran Khan \\ Masters of Applied Science \\ Graduate Department of Mechanical and Industrial Engineering \\ University of Toronto
}

2013

\begin{abstract}
This thesis describes the development of a MEMS repulsive actuator capable of producing a large out-of-plane force. Existing MEMS repulsive actuators are low out-of-plane force actuators that are unable to support or lift a mass of $1 \mathrm{mg}$. A high force MEMS repulsive actuator was developed to overcome this limitation. The design was optimized employing parameters of the actuator's fingers to increase the out-of-plane force. A design was developed based on the analytical results derived from extending the mathematical model of an existing actuator. A commercial manufacturing process, PolyMUMPs, was used to fabricate a prototype which was tested to validate the analytical and computational results. The prototype achieved an out-of-plane displacement of $15 \mu \mathrm{m}$ and a $0.2^{\circ}$ angular rotation. The resonance frequency was $120 \mathrm{~Hz}$, and the rise and fall times were measured as $14.5 \mathrm{~ms}$ and $3625 \mathrm{~ms}$ (3.6 sec), respectively. The estimated out-of-plane force is $40 \mu \mathrm{N}$.
\end{abstract}




\section{ACKNOWLEDGEMENT}

I am highly grateful to my supervisor, Prof. Ridha Ben Mrad, for his kind support, motivation and practical advice throughout this project. His continuous guidance and moral support helped me fulfil the requirements of my M.A.Sc program.

I would also like to thank Imed Zine-El-Abidine and CMC Microsystems for the technical help and support during the development of the actuator chip.

I would also like to thanks my fellow graduate students at the Mechatronics and Microsystem Design Laboratory (MMDL), Mike Schertzer, Vainatey Kulkarni, James Chong, Alaeddin Bani Milhim, Faez Ba Tis, Khalil Zahrr, and Bing Zhang for their expert help and advice.

I would also like to thanks my parents for their continual support and encouragement which enables me to achieve everything in my life. Finally, I would like to thank my wife Farah, and my sons Omer and Abdullah, for all their support, understanding, and appreciation. 


\section{Table of Contents}

\section{Chapter 1}

Introduction.......................................................

1.1.Motivation..........................................................

1.2.Literature Review.................................................

1.2.1. Thermal Actuators...............................................

1.2.2. Electromagnetic Actuators....................................4

1.2.3. Thin-film Metallic Glass Actuators...............................5

1.2.4. Electrostatic Actuators...........................................5

1.3. Research Objectives............................................8

1.4. List of Contributions.............................................. 8

1.5. Thesis outline ................................................... 9

2. Chapter 2 Design Analysis......................................... 10

2.1.Analytical Model................................................13

2.1.1. Rotational Stiffness of Anchor Springs........................16

2.1.2. Optimization Using Analytical Model.........................17

2.2. 3D Model of MEMS Repulsive Actuator ............................20

2.2.1. MEMS Repulsive Actuator with Payload Platform................22

2.3. Stiffness of the Payload Platform................................23

2.3.1. Design of a Supported Beam.................................24

2.3.2. Thickness of the MEMS Actuator............................24

2.3.3. Holding Platform with Electrostatic Springs...................25

2.4. Simulation Results of MEMS Actuator with Payload.................26

2.5. Simulation Results of MEMS Actuators under the Load Condition...27

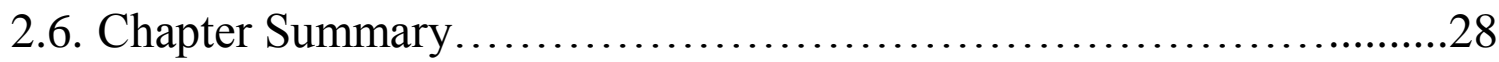

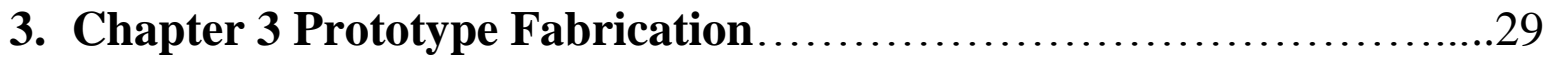


3.1. Double-layer Actuator...........................................29

3.2. Designing of a Supported Beam with Shield Layer....................31

3.3. Chapter Summary ............................................34

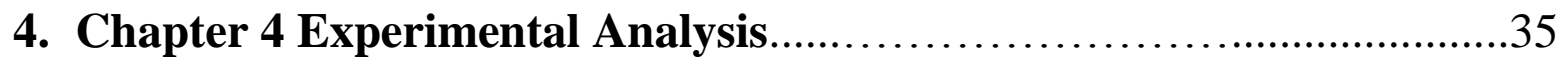

4.1. Experimental Setup........................................... 36

4.2. Static Measurements............................................37

4.2.1. Translational Measurements..................................37

4.2.2. Rotational Measurements using Position Sensor Diode...........39

4.2.3. Rotational Measurements under the Zygo Profilometer...........42

4.3. Dynamic Measurements...........................................44

4.3.1. Time Response..........................................44

4.3.2. Frequency Response.........................................47

4.4. Testing under the Load Conditions. ................................49

4.4.1. Procedure for Load Test....................................49

4.4.2. Expected Experimental Result with Load...........................50

4.5. Short Circuit Analysis..............................................51

4.6. Chapter Summary...............................................52

5. Chapter 5 Conclusions and Recommendations.......................53

5.1. Limitations of Current Design.......................................54

5.2. Recommendations to Improve the Design............................54

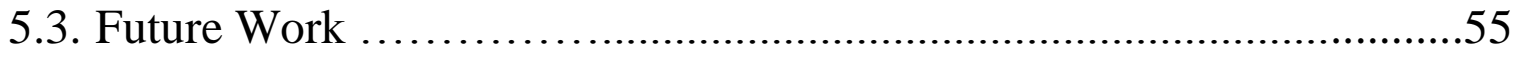

References...................................................................56

Appendix A Mask Layout of Prototype......................................59

Appendix B Development Process of Prototype in PolyMUMPs................61 Appendix C Other Design Options...............................................62 


\section{List of Tables}

Table 4-1 Electrical measurements of the chip before applying the voltage...... 35

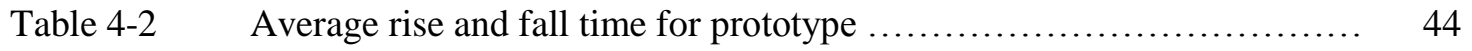

Table 4-3 Chip circuit measurement after short circuit..................... 51 


\section{List of Figures}

Figure 1-1 Control valve for micro and meso fluid channels. 2

Figure 1-2 Electrostatic attraction force between two plates of opposite charges. 6

Figure 1-3 MEMS repulsive actuator (a) Cross-section view of one unit (b) side view of 7 the moving finger.

$\begin{array}{lll}\text { Figure 2-1 Top view of MEMS repulsive actuator. } & 10\end{array}$

Figure 2-2 Proposed size of the overall device 12

Figure 2-3 Side view of a finger being repelled. 13

Figure 2-4 Dimension of an anchor spring. 16

Figure 2-5 Rotational stiffness of anchor springs (a) 3D model (b) Simulation result 17 under the applied couple.

Figure 2-6 Optimization of finger width at set value of moving finger length. 18

Figure 2-7 Optimization of moving finger length at optimized value of finger width. 18

Figure 2-8 Comparison the optimized value of finger width at $1400 \mu \mathrm{m}$ and $1800 \mu \mathrm{m} \quad 19$ finger length.

Figure 2-9 MEMS repulsive actuator (a) 3D Model in Coventor (b) Simulation result at 21 $75 \mathrm{~V}$.

Figure 2-10 Out-of-plane angular displacement as function of the applied voltage 21

Figure 2-11 Comparison between analytical and simulation results. 22

Figure 2-12 Complete model (a) 3D in Coventor (b) Simulation result at $100 \mathrm{~V}$. 23

Figure 2-13 Simulation result under load condition. 23

Figure 2-14 Simulation results (a) of single actuator with supporting beam (b) The 24 Completed model under load $10 \mu \mathrm{N}$.

Figure 2-15 Simulation results when subject to a load of $10 \mu \mathrm{N}$ (a) $10 \mu \mathrm{m}$ thickness (b) 25 $3.5 \mu \mathrm{m}$ thickness.

Figure 2-16 Electrostatic springs (a) 3D models (b) Simulation result with payload 26 platform.

Figure 2-17 Simulation results at $100 \mathrm{~V}$ (a) Translation displacement (b) Angular 27 rotations when only one actuator is actuated.

Figure 2-18 Payload platform under the load conditions. 27

Figure 3-1 Side view of a double-layer MEMS repulsive actuator. 29

Figure 3-2 Top view of (a) Single actuator (b) Exploded top view of fingertip showing 30 structural layer

Figure 3-3 Width of each poly layers for anchor springs. 31

Figure 3-4 Shield-layer effect (a) Attractive force between fixed and moving finger (b) 32 No attractive force between fixed and moving finger (Poly2) under the shield 
layer of Poly1.

Figure 3-5 Top view of moving and fixed fingers with shield layer. 33

Figure 3-6 3D view of a one unit cell moving and fixed fingers with shield layer (a) 33 shield layer on connected beam (b) Bottom view of connected beam and shield layer (c) Moving finger (Poly1) and shield layer (Poly1) (d) Moving finger with Poly2 and Poly1.

Figure 4-1 Prototype (a) packaged chip (b) Top view of in 2D drawing. 36

Figure 4-2 Chip inspection under the Zygo profilometer 37

Figure 4-3 2D and 3D views and surface profile across $\mathrm{AB}$ under the Zygo Profilometer 38 (a) At $0 \mathrm{~V}$ (b) at 120.

Figure 4-4 Out-of-plane displacement of the platform $0 \mathrm{~V} \sim 120 \mathrm{~V}$.

Figure 4-5 Experimental setup for angular measurement (b) 3D view of setup with laser 40 alignment.

Figure 4-6 Angular displacement based on the displacement of the laser spot in (a) X direction (b) Y direction.

Figure 4-7 Rotation of the platform in 2D view under the Zygo Profilometer, when 120 $\mathrm{V}$ is applied on (a) West actuator (b) East actuator (c) North actuator. (d) Surface profile along XX, shows the out-of-plane displacement of the north actuator about $\mathrm{CC}$ axis.

Figure 4-8 Angular rotation of the platform due to each actuator under the zygo profilometer.

Figure 4-9 Time responses $0 \mathrm{~V} \sim 160 \mathrm{~V}$ (a) Rise time in ms (b) Fall time in seconds. 45

Figure 4-10. Time responses for all actuators acting together $0 \mathrm{~V} \sim 160 \mathrm{~V}$ (a) Rise time in 46 milliseconds (b) Fall time in seconds.

Figure 4-11. Time responses $0 \mathrm{~V} \sim 160 \mathrm{~V}$ (a) Rise time in milliseconds (b) Fall time in 47 seconds.

Figure 4-12. Frequency response under the voltage of $0 \mathrm{~V} \sim 160 \mathrm{~V}$ (a) East actuator

(b)West actuator (c) South actuator (d) North actuator. 


\section{Chapter 1}

\section{Introduction}

\subsection{Motivation}

Micro-electro-mechanical-systems (MEMS) are used in industry in many applications such as for sensing and actuating. MEMS actuators are generally based on silicon substrates and developed through surface micromachining or bulk machining processes. They include features in micrometers to few millimetres in size. Examples of MEMS devices are micromirrors and tunable capacitors.

In the future, MEMS devices are expected to be used in more applications. These applications include manipulating a micro lens in MOEMS (Micro-Opto-Electro Mechanical System) [1], controlling flow in fluid channels [2], autofocus in phone cameras [3], and lifting and placing a micro object during micro-assembly operations [4]. MEMS actuators with large out-of-plane motion are more suitable for these interactive tasks. In all the above applications, large out-ofplane force is required to move a mass of $1 \mathrm{mg}$ to $5 \mathrm{mg}$ with strokes ranging from $10 \mu \mathrm{m}$ to 100 $\mu \mathrm{m}$. The detail of these applications is described below.

\section{Micro-Opto-Electro Mechanical Systems}

Out-of-plane MEMS actuators are in great demand in MOEMS in order to manipulate micro optical components such as micro lenses, micro optical benches and fiber optic arrays. The alignment of a fibre optic cable with a micro lens is of great challenge in telecommunication systems [5]. MEMS grippers are required to hold and place micro optical components precisely. A number of linear micro actuators, electrothermal [6], electromagnetic [7-8], electrostatic [9- 
10] have been demonstrated in MOEMS. The performance of these actuators has been limited [6-10].

\section{Flow Control Valves}

Out-of-plane MEMS actuators are also very useful in controlling the flow of fluids in micro and meso channels. An application of an out-of-plane MEMS actuator to control a valve is discussed in [2] in which micro bellows were designed based on a MEMS technology. The actuator expands in a direction away from the substrate plane, as shown in Figure 1-1. The micro bellows apply a force to the ramp thereby causing rotation of the ramp in a direction away from the substrate plane.

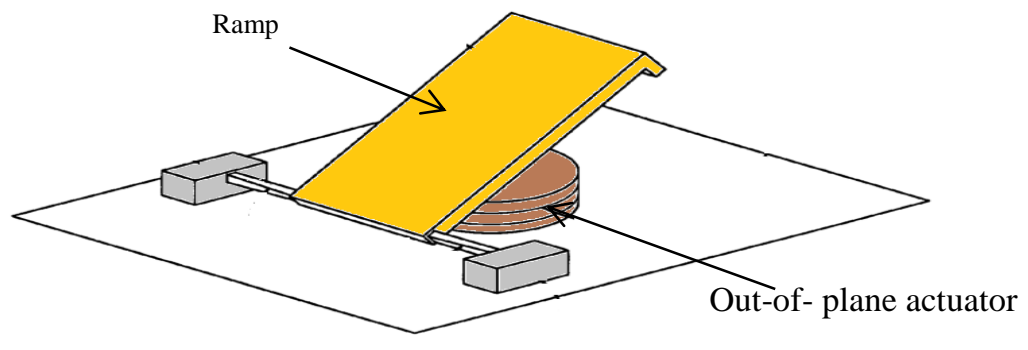

Figure 1-1. Control valve for micro and meso fluid channels [2]

\section{Micro Assembly Operations}

In the micro assembly process, micron-scale components are positioned, oriented, bonded and assembled to achieve functional assemblies. A MEMS out-of plane actuator is used to assemble the micro-components. The current setup of assembly is not fully automated and unable to provide precise adjustments of the components. A MEMS-based thermal actuator in [11] and a silicon micro fabricated pusher tool in [4] were used to perform limited tasks to achieve in-plane assembly operations. 


\section{Autofocus in Phone Cameras}

Out-of-plane MEMS actuators have great potential for use in future phone cameras for autofocusing [12-15]. Current phone cameras use less efficient autofocus and image stabilization technologies. Voice coil motors (VCM) are a commercially available technology for autofocus in phone cameras. A VCM has a large thickness and high power consumption, which limits its use in future phone cameras [16]. The mass of the optical lens for future phone cameras is anticipated to be $1 \mathrm{mg}$ to $5 \mathrm{mg}$ while the overall thickness of a cell phone is limited to $4 \mathrm{~mm}$. To date, different technologies other than MEMS [17-19] have been tested in research for new autofocus actuators for phone cameras, but their performance has been limited. As a result, a thin out-of-plane MEMS actuator with large out-of-plane force is still needed for this application.

\subsection{Literature Review}

Researchers are pursuing different solutions to develop large out-of-plane force and displacement actuators based on MEMS technology to fulfil the demand of the applications listed above. The details of the actuators developed so far are given below.

\subsubsection{Thermal Actuators}

Bimorph and Electrothermal actuators are the most common thermal actuators for producing out-of-plane motion. Thermal bimorph actuators [20] consist of two materials with equal lengths but with different coefficients of thermal expansion. These materials are joined along their longitudinal axis. When the actuator is subject to an increase in temperature each layer deforms 
differently resulting in the beam curving towards the layer with a lower coefficient of thermal expansion. The direction of out-of-plane motion can be controlled by changing the arrangement of the materials.

Electrothermal actuators develop out-of-plane motion by inducing joule heating in a MEMS device through the application of a current. One such actuator can generate an out-of-plane motion of $30 \mu \mathrm{m}$ with an applied voltage of $5 \mathrm{~V}$ [21]. The design is based on a single layer of an SOI (Silicon on Insulator) wafer and two notches near the middle of the actuator beams. Although electrothermal actuators are capable of producing large output force and displacement, they require large currents which create limitations in a number of applications.

\subsubsection{Electromagnetic Actuators}

A magnetic field can be used to produce force, torque and displacement [22] based on different magnetic actuation principles. A driving magnetic field requires current carrying wires, inductor coils, and/or magnets.

A bidirectional cantilever magnetic actuator was developed in [23]. In this design, an array of permanent magnets are electroplated at the tip of a silicon cantilever beam in order to produce vertical actuation. The cantilever beam is $6 \mathrm{~mm}$ long, $1 \mathrm{~mm}$ wide, and $13 \mu \mathrm{m}$ thick. A commercial inductor is used as the electromagnet to drive the bidirectional actuator. The magnetic force applied to the cantilever is

$\mathrm{F}=\mathrm{V} \cdot \mathrm{M}_{\mathrm{s}} \frac{\partial \mathrm{H}}{\partial \mathrm{Z}}$ 
where $V$ is the volume of a magnetic element, $M_{S}$ is the magnetization of that magnet, and $\frac{\partial H}{\partial Z}$ is the change in magnetic field with respect to vertical distance. An input current of $100 \mathrm{~mA}$ resulted in an out-of-plane force of $50 \mu \mathrm{N}$ and a displacement of $88 \mu \mathrm{m}$.

Electromagnetic MEMS actuators are potentially capable of large out-of-plane force and displacement. But the size of the magnetic core needs to be large, and a high amount of current is also required, which is not suitable for many sensitive applications such as robotic surgery or autofocus in phone cameras.

\subsubsection{Thin-film Metallic Glass Actuators}

Thin film metallic glass (TFMG) is a new MEMS material [24]. A TFMG actuator is used to develop conical spring moving electrodes which provide out-of-plane motion and large output force. A single device using an array of 100 micro actuators can produce out-of-plane stroke of $200 \mu \mathrm{m}$ with output force $1 \mathrm{mN}$ (where the size of each actuator is $1 \mathrm{~mm}^{2}$ ) [24]. Fabrication of these devices is not simple and requires a process that involves sputtering, lift-off patterning, and sacrificial layer etching.

\subsubsection{Electrostatic Actuators}

A conventional out-of-plane electrostatic actuator has a parallel plate structure consisting of fixed and moving electrode plates. When a voltage is applied, an electrostatic attractive force pulls the moving electrode down towards the fixed electrode. The motion produces an out-ofplane direction due to the attractive force as shown in Figure 1-2. The amount of force is given by 


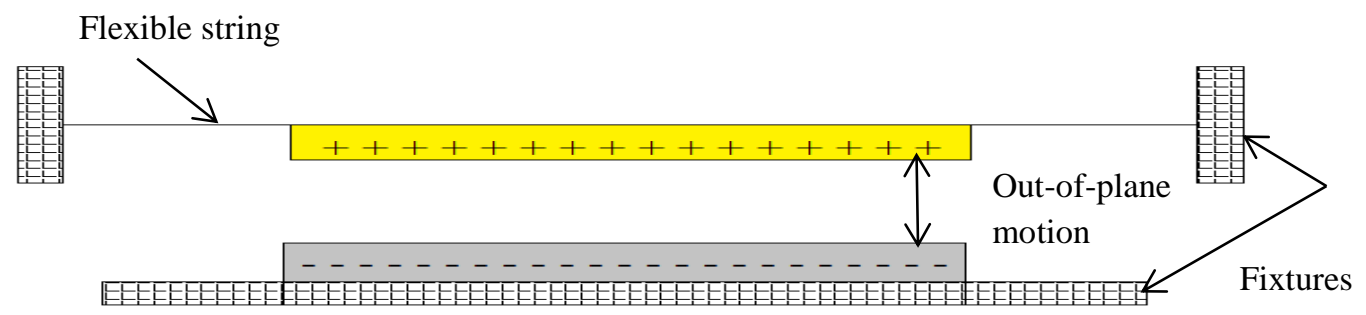

Figure 1-2. Electrostatic attraction force between two plates of opposite charges

$$
F=\frac{1}{2} \frac{V^{2} \varepsilon \cdot A}{(g)^{2}}
$$

where $V$ is the applied voltage, $g$ is the gap distance between the fixed and moving plate, $\varepsilon$ is the vacuum dielectric constant, and $A$ is the two plates overlapping area [20].

In these devices, the output force is a non-linear function of the gap distance. This non-linearity results in the so-called pull-in effect where the moving plate sticks to the fixed plate when the displacement of the moving electrode exceeds one-third of the initial distance. Beyond this distance, the electrostatic force increases very rapidly and does not allow the restoring force to move the plate up.

A large number of electrostatic actuators are developed to avoid the pull-in effect [25-36]. In which, a MEMS repulsive actuator has been developed to avoid the pull-in effect completely while delivering large out-of-plane stroke [25-31]. This actuator consists of fixed and moveable fingers. Fixed fingers are further classified in aligned and unaligned fixed fingers. If the voltage is applied in a manner as shown in the cross-section view in Figure 1-3 (a), then the combined effect of the electrostatic field produces an out-of-plane force to move the moveable finger outof-plane direction. The side view of the unit cell is also shown in Figure 1-3 (b). A stroke of 80 $\mu \mathrm{m}$ reported in [26] is the maximum stroke achieved by any out-of-plane electrostatic actuators. 


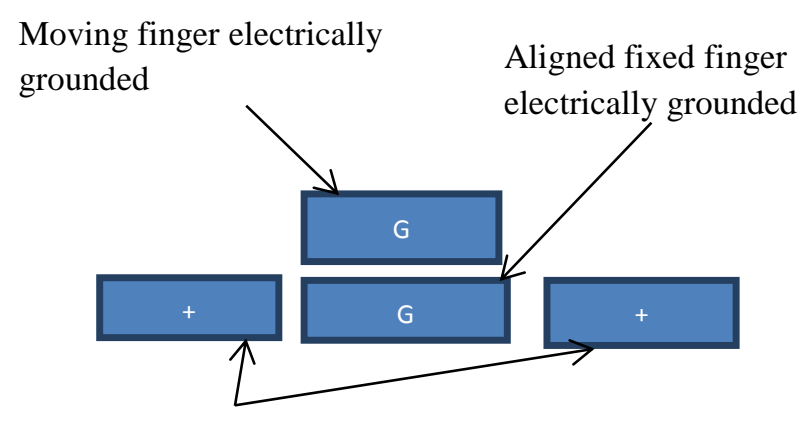

Unaligned fixed finger with electric potential

(a)

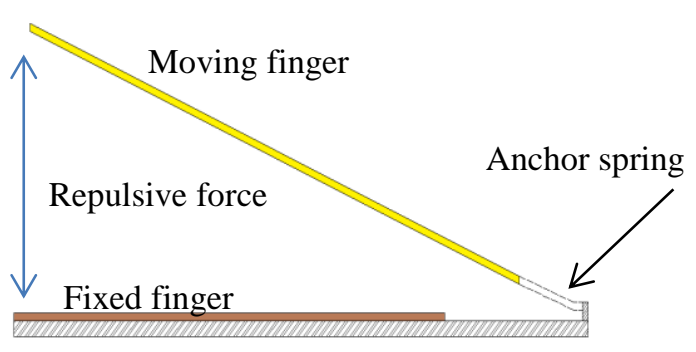

(b)

Figure 1-3. (a) Cross-section view of one unit (b) side view of the moving finger

Although the MEMS repulsive actuator can produce a high stroke, it has a very low out-of-plane force especially at large stroke, which is not sufficient for commercial applications. The out-ofplane force can be estimated from [27] which is only $0.8 \mu \mathrm{N}$ at $100 \mathrm{~V}$.

A MEMS repulsive actuator has many advantages such as small size, a large out-of-plane stroke, low power consumption, fast response time, small hysteresis, ease of manufacture through surface micromachining processes, and compatibility with integrated circuits. This actuator has great potential for use in various applications. If the out-of-plane force could be increased in order to support and manipulate masses of $1 \mathrm{mg}$ or more with reasonable stroke length, it would become commercially viable.

This thesis will report on contributions focusing on the development of a MEMS repulsive actuator offering the following features:

- Ability to lift a mass of $1 \mathrm{mg}$ or more against gravitational acceleration.

- Compatibility with commercially available surface micromachining processes.

- Ability to produce $20 \mu \mathrm{m}$ out-of-plane stroke when carrying a load. 


\subsection{Research Objectives}

1. Develop a MEMS repulsive actuator able to produce an out-of-plane force of $10 \mu \mathrm{N}$.

2. Develop a high-stiffness payload platform to hold the mass while integrating the actuators.

3. Fabricate a prototype based on a commercial MEMS manufacturing process.

4. Test the static and dynamic characteristics of the prototype.

\subsection{List of Contributions}

The goal of this thesis project was to re-design the MEMS repulsive actuator to increase the output force for lifting an object of a mass of $1 \mathrm{mg}$ or more.

This work makes the following contributions:

- A MEMS repulsive actuator was redesigned to produce large out-of-plane force. The design parameters are optimized to achieve a large force.

- The stiffness of the payload platform was increased by incorporating support beams and electrostatic springs compatible with the PolyMUMPs process.

- A prototype was developed based on a commercial PolyMUMPs process.

- The static and dynamic performance of the prototype was tested. 


\subsection{Thesis outline}

\section{Chapter 2: Design Analysis}

This chapter describes the design concepts and detailed analysis of the MEMS repulsive actuator and associated holding platform. An analytical model was used to optimize the performance of the actuator. The mathematical computational results are also discussed. This chapter also describes different options for increasing the stiffness of the payload platform.

\section{Chapter 3: Prototype Fabrication}

This chapter describes the PolyMUMPs process used to fabricate the prototype of the final proposed design. The design rules and the limitations of the fabrication process are discussed.

\section{Chapter 4: Experimental Analysis}

This chapter presents the results of the static and dynamic performance tests conducted on the prototype. The differences between the experimental and simulation results are also discussed.

\section{Chapter 5: Conclusions}

This chapter describes the overall research contributions, prototype features, possible limitations and future recommendations. 


\section{Chapter 2}

\section{Design Analysis}

This chapter reviews the design analysis of a MEMS repulsive actuator with large out-of-plane force and stroke. It presents the design of an anchor spring and the optimization of the length and width of the attached moving finger using an analytical model.

A MEMS repulsive actuator includes a set of finger electrodes. These fingers can be classified into fixed and moving fingers. Fixed fingers are further divided into aligned and unaligned fixed fingers as shown in Figure 2-1. For rotational motion, moving fingers are connected to a common rail, which are then suspended by the anchor springs. By applying the voltage to these finger electrodes an out-of-plane displacement is achieved.

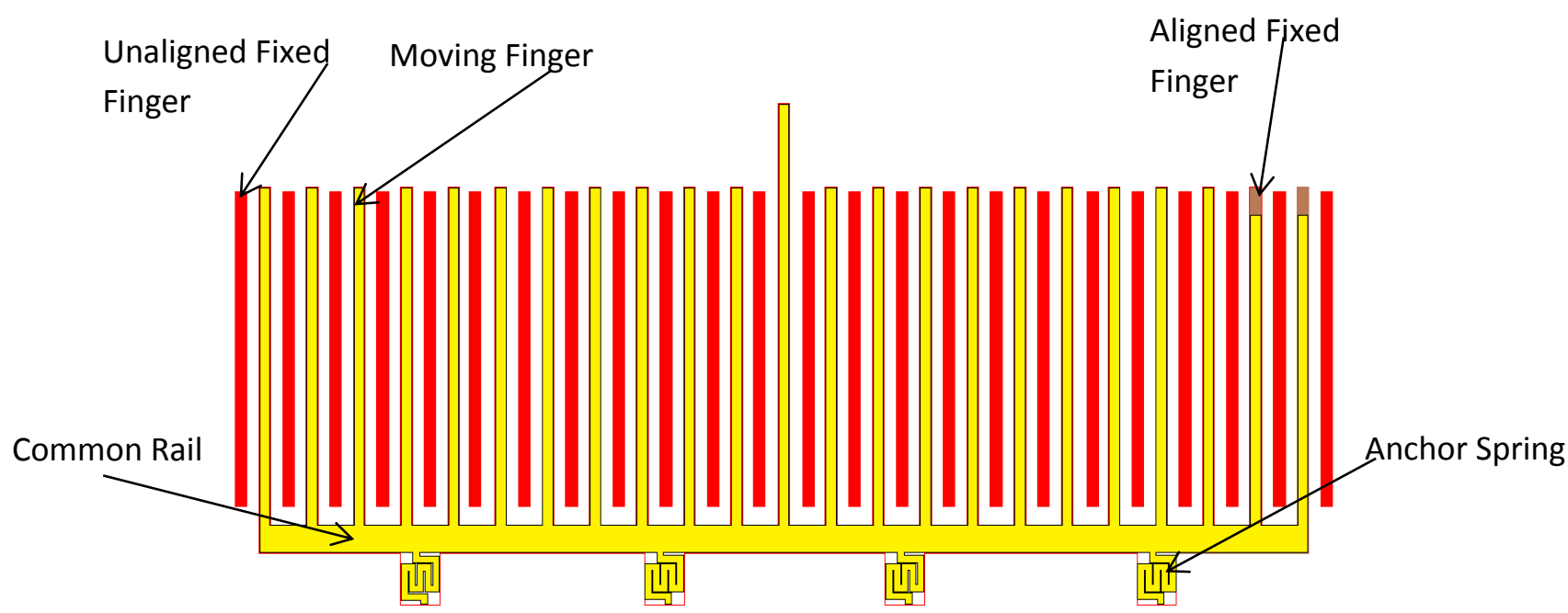

Figure 2-1. Top view of MEMS Repulsive Actuator.

Existing MEMS repulsive actuators [25-27] are low out-of-plane force actuators. In order for these devices to be adapted for a wider range of practical applications, they need to be re- 
designed to provide an out-of-plane force of $10 \mu \mathrm{N}$ or more. The output force of a MEMS repulsive actuator is given by:

$$
F=\frac{\mathrm{N} \cdot V^{2} \cdot L}{g} \cdot \frac{d C}{d h},
$$

where $N$ is the number of moving fingers, $V$ is the applied voltage, $L$ is the length of the moving fingers, $g$ is the width of the fixed and moving fingers, and $\frac{d C}{d h}$ is the differential ratio between the capacitance and the initial gap distance between the fixed and moveable fingers [26]. The stroke length is determined by $\frac{d C}{d h}$. The capacitance between the unaligned fixed fingers and the remaining fingers within the given range increases when the moving fingers move away from the fixed fingers. The out-of-plane force is proportional to the number of fingers and moving fingers length, and is also inversely proportional to the finger width.

Optimization of the proposed actuator can be achieved by performing a parametric study of the variables in Equation 2.1. The number of fingers can be increased by reducing the finger width of the actuator or increasing the total width of the actuator. However, reducing the finger width will reduce the stroke length [26]. Once the gap distance between the fixed and moving finger increases in comparison to the width of the finger, then out-of-plane repulsive force converts to the attractive force. Large displacement can be achieved at a large width of the finger. Increasing the width of the actuator to add more fingers does not compromise the stroke length, but instead, the footprint of the device.

The length of the fingers can be set as large as possible with respect to the dimension of the actuator, to increase the output force. In the following analysis, the width of a single actuator is held constant at $5.3 \mathrm{~mm}$ and the length of the actuator varies between $1.2 \mathrm{~mm}$ to $2 \mathrm{~mm}$, 
depending on the footprint of the device. The total size of the proposed device consists of four actuators and a platform for translating and rotating a payload of $1 \mathrm{mg}$, is $10 \mathrm{~mm} \times 10 \mathrm{~mm}$ with a thickness of $6 \mu \mathrm{m}$ (Figure 2-2).

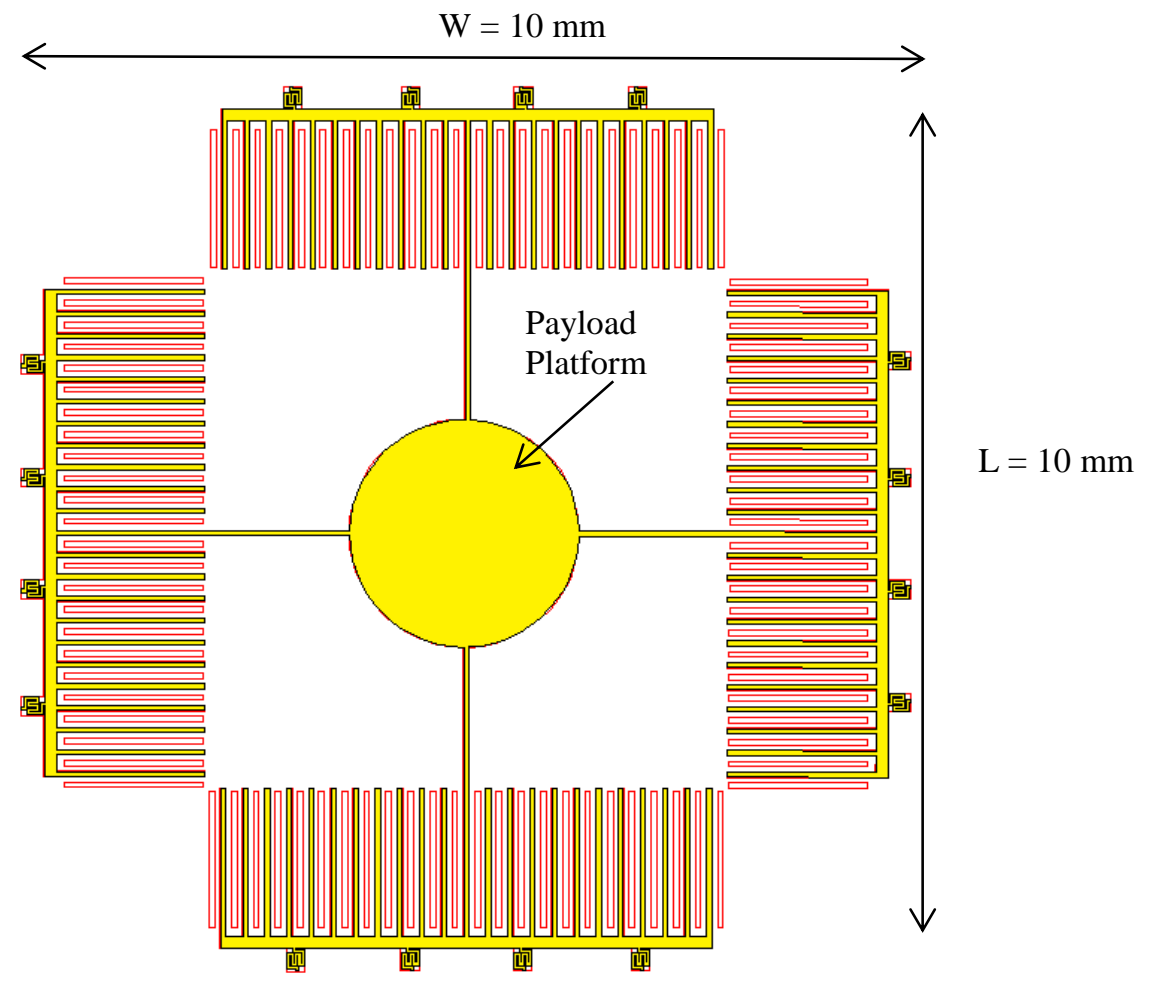

Figure 2-2. Proposed size of the overall device.

The length of the finger was initially assumed to be $1400 \mu \mathrm{m}$. The performance of the proposed actuator was initially optimized by varying the number of fingers, to determine the finger width for large out-of-plane force and displacement. The finger length was then varied to increase the output force without compromising the stroke length. An analytical model [25] was employed to search for the best values of the finger width and moving finger length. 


\subsection{Analytical Model}

A side view of a MEMS repulsive actuator is shown in Figure 2-3. Under an applied voltage, an electrostatic torque is developed to produce angular displacement. At some point, the electrostatic torque is balanced by the restoring torque.

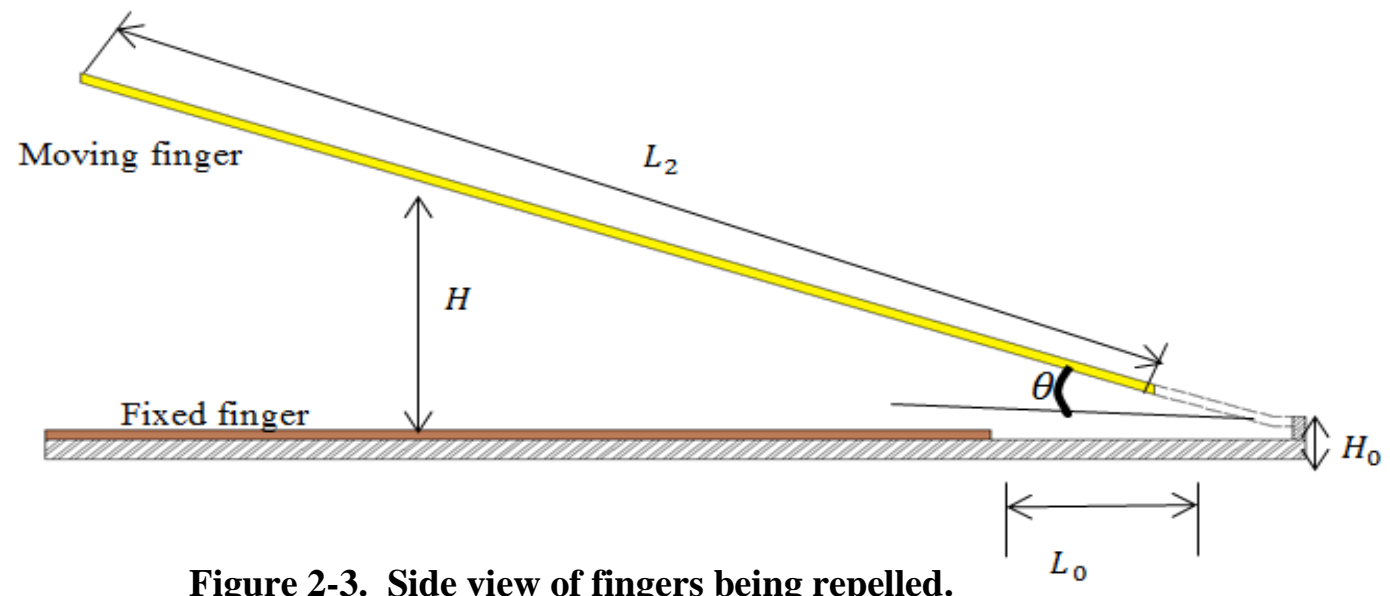

Figure 2-3. Side view of fingers being repelled.

For an odd number of moving fingers, an analytical model was devised in [25] to determine the output torque of the actuator. The torque was estimated for a half portion of the actuator (from the central finger to the first finger), as follows:

$$
T_{e}(\text { Half })=V^{2} \cos \theta \sum_{k=1}^{N_{\text {half }}} \sum_{i=0}^{7} \frac{a_{i}}{g^{i+1}} C(k) \int_{L_{0} \sec \theta}^{L_{2}} l .\left(l \cdot \sin \theta+H_{0}\right)^{i} \cdot d l
$$

The remaining half portion of the actuator is symmetrical to the first. The total torque can then be calculated by multiplying Equation 2.2 by 2 :

$$
T_{e}=2 V^{2} \cos \theta \sum_{k=1}^{N_{\text {half }}} \sum_{i=0}^{7} \frac{a_{i}}{g^{i+1}} C(k) \int_{L_{0} \sec \theta}^{L_{2}} l \cdot\left(l \cdot \sin \theta+H_{0}\right)^{i} \cdot d l
$$


where $T_{e}$ is the total electrostatic torque, $g$ is the finger width, $V$ is the applied voltage, $\theta$ is the angular displacement of a moving finger, $k$ is the index number, $N_{\text {half }}$ equals half of the number of fingers, and $a_{i}(i=[0-7])$ are the following coefficients:

$$
\begin{gathered}
a_{o}=0.38019 \times 10^{-11}, \quad a_{1}=0.74351 \times 10^{-11}, \quad a_{2}=0.54809 \times 10^{-11}, \\
a_{3}=-0.22101 \times 10^{-11}, a_{4}=0.53711 \times 10^{-12}, a_{5}=-0.78854 \times 10^{-13}, \\
a_{6}=0.64592 \times 10^{-14}, \quad a_{7}=-0.22687 \times 10^{-15} .
\end{gathered}
$$

$L_{2}$ is the length of the moving finger, $L_{0}$ is the constant in-plane distance between the fixed and moving fingers and $H_{o}$ is the initial vertical gap between the fixed and moving fingers (Figure 2-3). Since the PolyMUMPs process was used to fabricate the actuator, $L_{0}$ and $H_{o}$ are set to be $150 \mu \mathrm{m}$ and $0.75 \mu \mathrm{m}$ (dimple height) [26]. A constant, $C(K)$ represents the contribution of each finger in producing a torque. Since the actuator has an odd number of fingers, the torque generated for the central finger is halved. A constant $C(K)$ is $\{1,1,1,1,0.5\}$ for an actuator with 9 moving fingers.

This model was developed based on the assumption that the finger width is much larger than the thickness of the finger. Therefore, the thickness of the fingers was considered negligible.

The static balance condition can be achieved when $T_{e}=T_{s}$

where $T_{S}$ is the spring restoring torque $\left(T_{S}=k_{\theta} \theta\right)$ :

$$
T_{e}=2 V^{2} \cos \theta \sum_{k=1}^{N_{\text {half }}} \sum_{i=0}^{7} \frac{a_{i}}{g^{i+1}} C(k) \int_{L_{0} \sec \theta}^{L_{2}} l .\left(l \cdot \sin \theta+H_{0}\right)^{i} \cdot d l=k_{\theta} \theta
$$




$$
2 V^{2} \cos \theta \sum_{k=1}^{N_{\text {half }}} \sum_{i=0}^{7} \frac{a_{i}}{g^{i+1}} C(k) \int_{L_{0} \sec \theta}^{L_{2}} l \cdot\left(l \cdot \sin \theta+H_{0}\right)^{i} \cdot d l-k_{\theta} \theta=0
$$

Equation 2.4 shows the static balanced condition of the actuator which can be used to determine the angular displacement of the moving fingers.

This equation can be used to optimize the width and length of the moving finger of the proposed actuator for maximum output displacement and force. The rotational stiffness $k_{\theta}$ is required for this analysis. Anchor springs are designed to meet the proposed actuator requirement. The rotational stiffness is calculated in the following subsection.

\subsubsection{Rotational Stiffness of an Anchor Springs}

In the model presented in [27], serpentine classic springs were used. Serpentine rotational springs are used in this design because they are less stiff in the out-of-plane direction so they should allow larger out-of-plane displacement. The stiffness of an anchor spring is dependent on its width, length, and number of turns. The minimum width of the springs is $34 \mu \mathrm{m}$ as constrained by the PolyMUMPs process. Similarly, an increase in the length of the springs decreases the stiffness, but the length is constrained by the overall footprint. The dimensions of the springs were chosen to fit within the available design area. The dimension of the anchor springs is shown in Figure 2-4. 


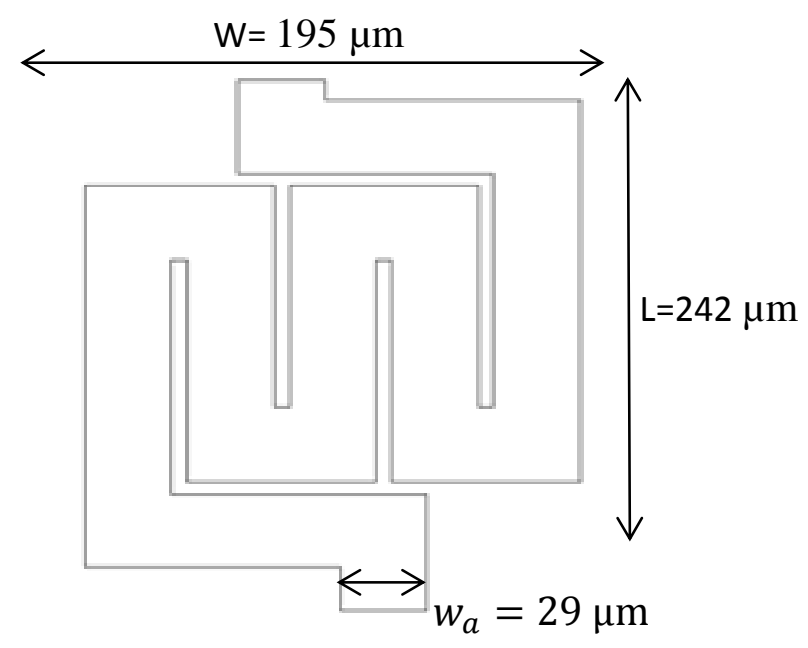

Figure 2-4. Dimension of an anchor spring.

Due to the large width of the actuator, four anchor springs are used to connect the actuator. The rotational stiffness of the anchor spring is computed using the software Coventor.

The equation for the restoring torque of the anchor spring is:

$$
T s=K_{\theta} \theta
$$

where $K_{\theta}$ is the rotational stiffness of the springs and $\theta$ is the angular displacement. For estimating the rotational stiffness, a single actuator was considered as a rigid beam with a width of $5300 \mu \mathrm{m}$, a length of $1400 \mu \mathrm{m}$, and a thickness of $2 \mu \mathrm{m}$. One end of the beam is connected to the anchored springs. A moment of $200 \mu \mathrm{N}$. $\mu \mathrm{m}$ was applied to simulate the angular displacement Figure 2-5(a). The Extruded Beam Algorithm was used to mesh the model. Simulation results are shown in Figure 2-5(b) which is independent of mesh size.

Rotational stiffness was computed to be $267 \times 10^{-9} \mathrm{~N} . \mathrm{m} / \mathrm{rad}$ by using Equation 2.5. In order to validate the result, different moments were applied at different places of the beam. The same value was achieved, which shows that the rotational stiffness value is independent of the size of the applied torque and the position of the applied torque. 


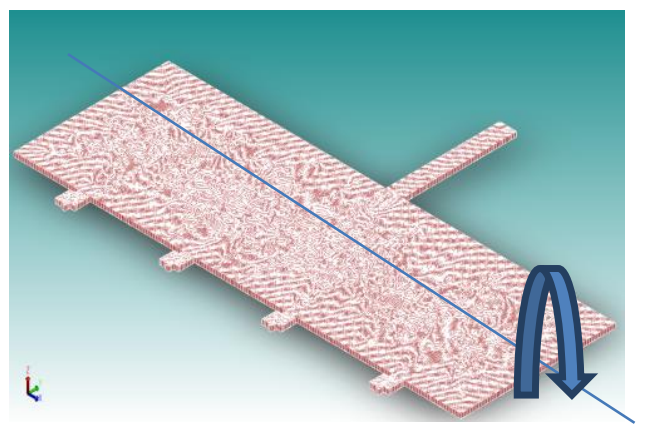

(a)

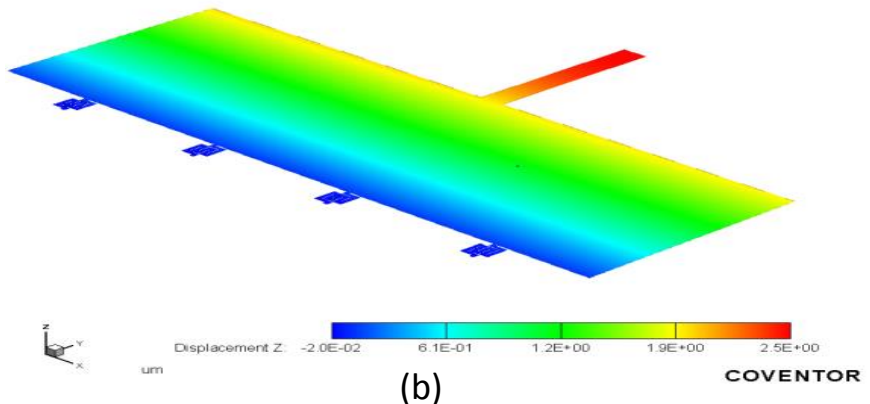

(b)

Figure 2-5. Rotational stiffness of anchor springs (a) 3D model (b) simulation result under the applied couple.

\subsubsection{Optimization Using an Analytical Model}

The finger width and moving finger length are optimized in this section to produce a large outof-plane displacement and force. The optimization procedure is based on the following three steps: In the first step, Equation (2.4) was used to determine actuator performance range over finger widths for a finger length of $1400 \mu \mathrm{m}$. Displacement was found to increase with finger width from $40-50 \mu \mathrm{m}$ before falling for a width greater than $75 \mu \mathrm{m}$ (Figure 2-6). In the useful range of the finger width, the out-of-plane angular displacement is $2.4^{\circ} \pm 0.01^{\circ}$. This suggests that useful range for the finger widths in this case would be 50-75 $\mu \mathrm{m}$, where the curve displays a local maximum. Since small finger width allows for a large number of fingers, the value of the finger width $(60 \mu \mathrm{m})$ in the useful range can be considered to be the best finger width for producing a large out-of-plane force. 


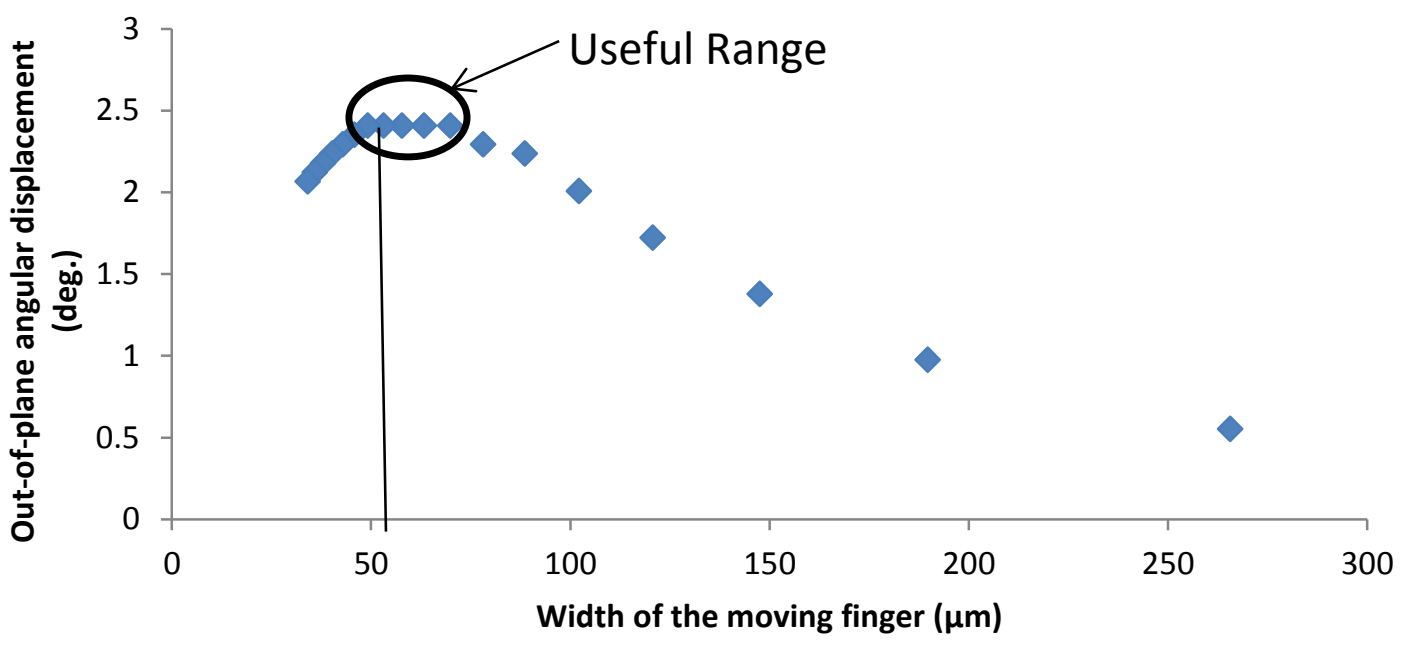

Figure 2-6. Optimization of finger width at a set value of moving finger length.

After determining a useful range for the finger width, a similar procedure was used to determine the effect of moving finger length on out-of-plane displacement (Figure 2-7). Out-of-plane displacement, again, shows a local maximum with respect to finger length. Here, the useful range is $1200 \mu \mathrm{m}$ to $1800 \mu \mathrm{m}$, where angular displacement is $2.45^{\circ} \pm 0.01^{\circ}$. Since the out-ofplane force is proportional to the moving finger length (Equation 2.1), the largest length value $(1800 \mu \mathrm{m})$ from the useful range is considered.

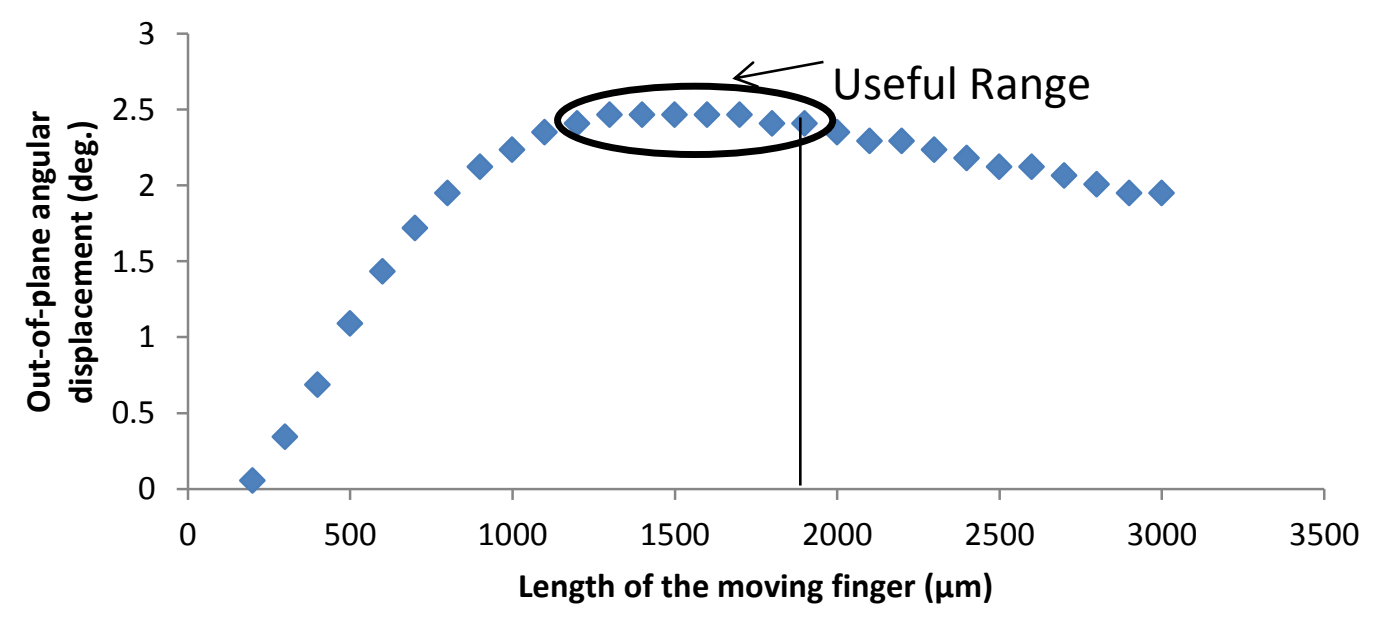

Figure 2-7. Optimization of moving finger length at optimized value of finger width. 
After optimizing finger length, the finger width was again analyzed at the considered length $(1800 \mu \mathrm{m})$. A comparison of the finger width analysis for length $1400 \mu \mathrm{m}$ and $1800 \mu \mathrm{m}$ is shown in Figure 2-8. The shape of the two curves is similar and the best finger width at length of $1800 \mu \mathrm{m}$ was again $60 \mu \mathrm{m}$. However, the deviation of both optimized curves is significant at large finger width values. The chosen finger width value $(60 \mu \mathrm{m})$ shows good correspondence for both values of finger length.

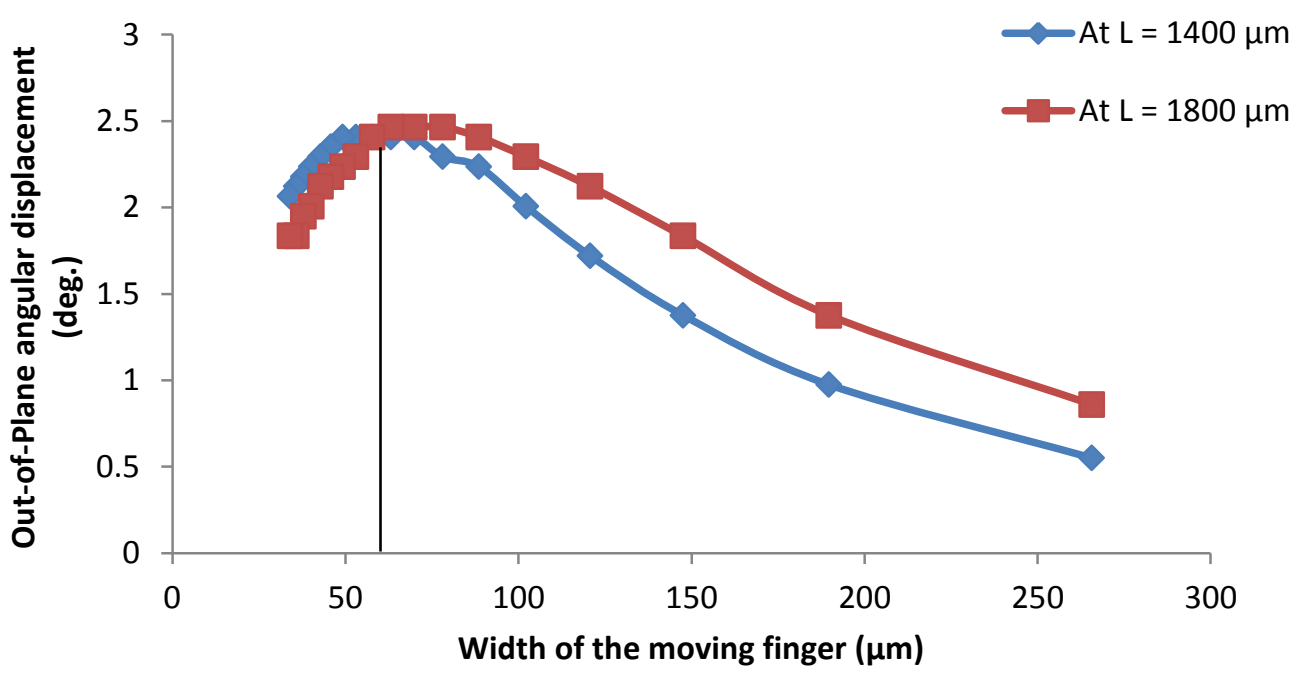

Figure 2-8. Comparison of the optimized value of finger width at $1400 \mu \mathrm{m}$ and $1800 \mu \mathrm{m}$ finger length.

In the useful range, the angular displacement is constant within $\pm 0.01^{\circ}$ with respect to length and width of the finger. This shows that the value of $\frac{d C}{d h}$ is constant (Equation 2.1). Therefore, the output force can be maximized by selecting a large finger length and the minimum finger width from the useful ranges (Figure 2-4 and Figure 2-5). The number of moving fingers that can be used based on the desired footprint is:

$$
\mathrm{W}=4 . N . g
$$


where $W$ is the width of the actuator fixed at $5.3 \mathrm{~mm}$. The calculated value of $N$ is 23 . Since the force $F$ is proportional to the number of fingers, a large number of fingers is required for a large out-of- plane force.

The proposed actuator is designed for large out-of-plane force; therefore, a large number of fingers are recommended. However, reduction in finger width is also limited due to the assumption of the analytical model. Further, a decrease in the finger width increases the ratio of the finger width and the finger thickness. In the development of the analytical model, finger thickness was considered negligible because of the small ratio. This assumption will not be applicable if the ratio of finger width and the finger thickness is increased. Therefore, the value chosen for $N$ (equal to 23) is reasonable for the proposed actuator.

\subsection{D Model of MEMS Repulsive Actuator}

A 2D single actuator based on the optimized result was developed in AutoCAD. A 3D model of the proposed actuator was developed in Coventor Software (Figure 2-9). Coventor simulates the electrical and mechanical systems by iterating between MemMech and MemElectro modules. The Extrude Bricks Meshing Algorithm was used to simulate the model. Simulation result with independent mesh size is shown in Figure 2-9(b). The out-of-plane force and displacement were computed to be $9.5 \mu \mathrm{N}$ and $35 \mu \mathrm{m}$, respectively at $75 \mathrm{~V}$. 


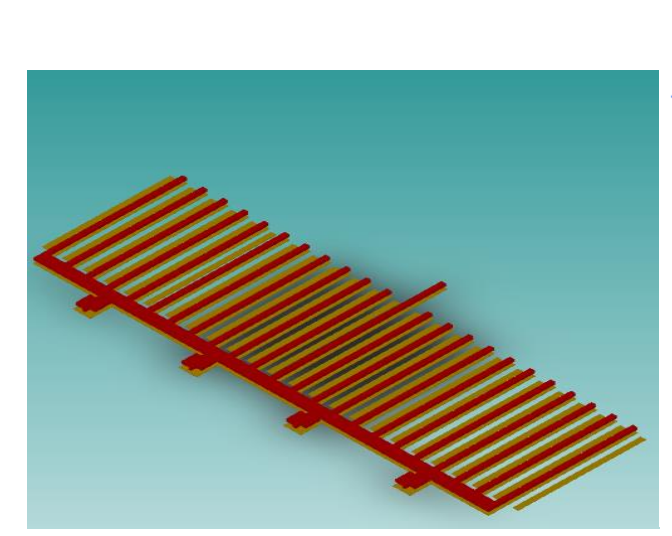

(a)

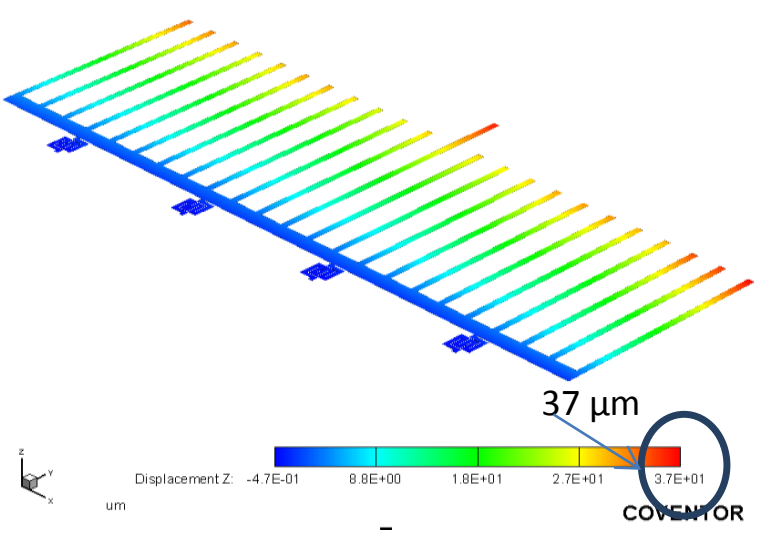

(b)

Figure 2-9. (a) 3D Model in Coventor (exaggerated in $\mathrm{Z}$ direction) (b) Simulation result of a single actuator at $75 \mathrm{~V}$.

Using Equation 2.4, the rotational angle can be calculated for specific input voltage as shown in Figure 2-10.

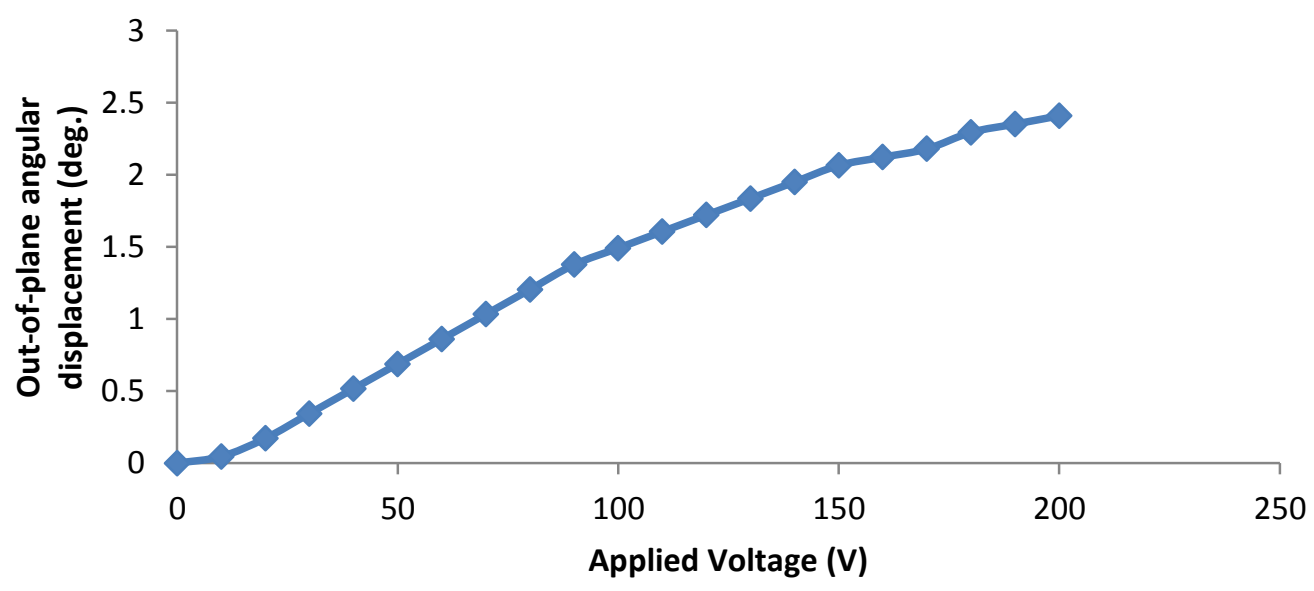

Figure 2-10. Out-of-plane angular displacement as function of the applied voltage.

This result is compared with a single actuator model that was simulated for applied voltages between $0 \mathrm{~V} \sim 80 \mathrm{~V}$. A good agreement between the analytical and simulation results, with percentage errors less than $10 \%$, is observed in the $0 \mathrm{~V} \sim 80 \mathrm{~V}$ range (Figure 2-11). It can also be concluded that the analytical model developed in [26] can be used to optimize the 
characteristics of large size of MEMS repulsive actuators, as long as the design was developed based on the assumptions of the analytical model. In the new design, out-of-plane force of $9.5 \mu \mathrm{N}$ is predicted at $75 \mathrm{~V}$. This improvement in force is over an order of magnitude when compared to $0.8 \mu \mathrm{N}$ at $100 \mathrm{~V}$ [27].

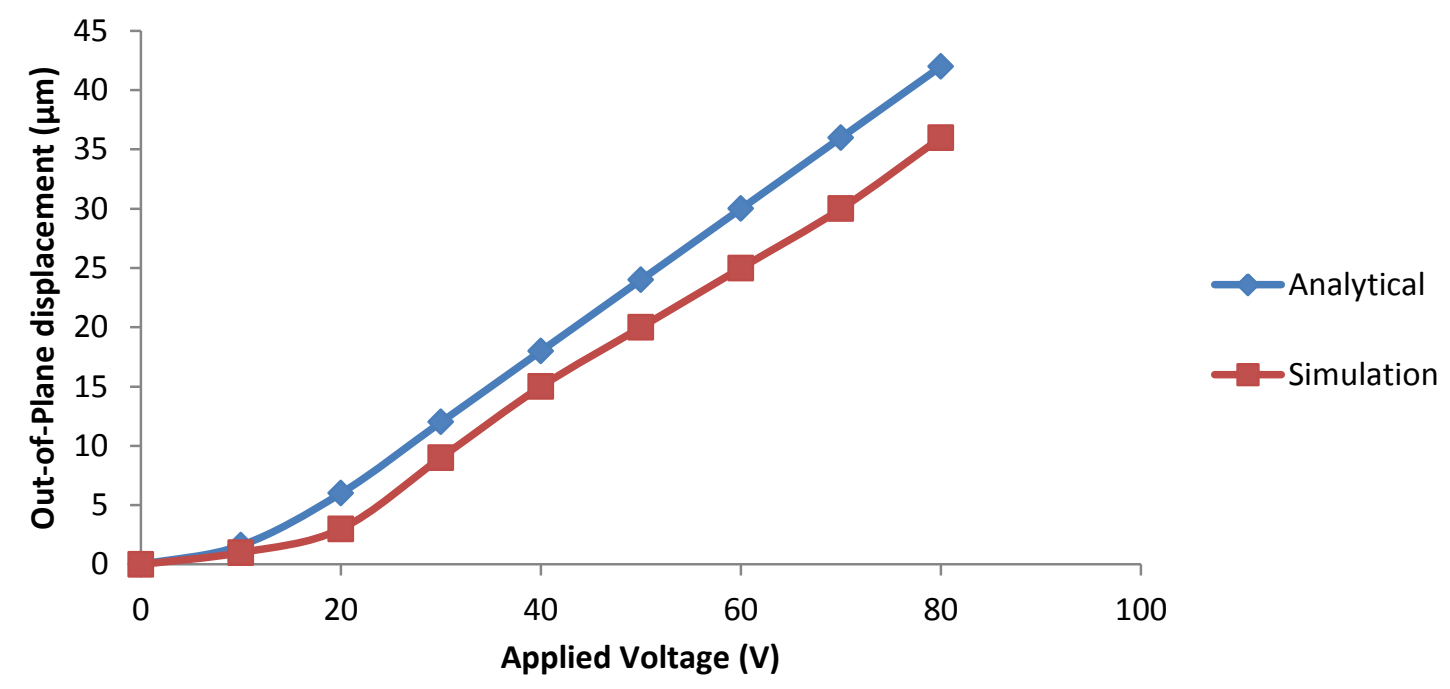

Figure 2-11. Comparison between analytical and simulation results.

\subsubsection{MEMS Repulsive Actuator with Payload Platform}

A payload platform is required to lift or hold the mass of $1 \mathrm{mg}$. The payload platform is designed to be manipulated by four single actuators as shown in figure 2-12 (a). The platform is $2.5 \mathrm{~mm}$ in diameter. The platform is connected to the actuators through a rigid beam. Simulation results of the complete model (Figure 2-12) predict that out-of-plane displacement of the payload platform is $25 \mu \mathrm{m}$ and the output force is $42 \mu \mathrm{N}$. 


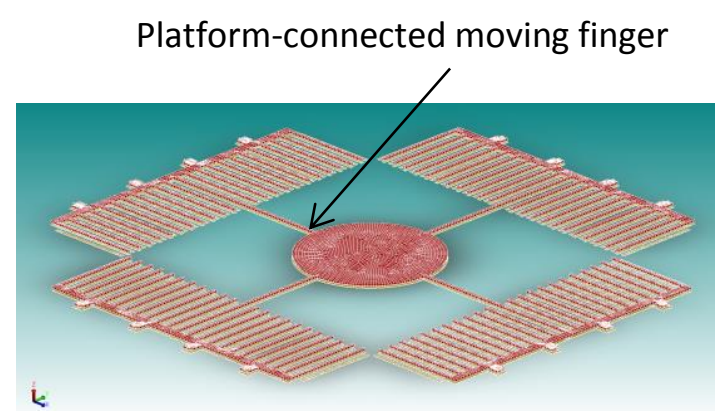

(a)

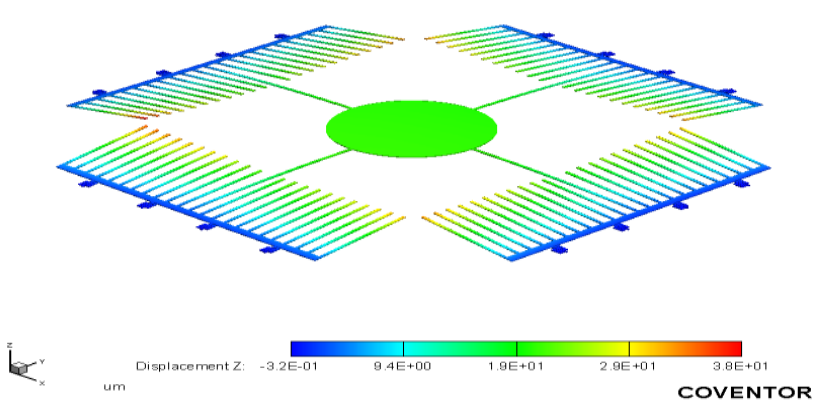

(b)

Figure 2-12. The Completed model (a) 3D in Coventor (b) Simulation result of complete model.

\subsection{Stiffness of the Payload Platform}

To examine the holding capability of the platform carrying a $1 \mathrm{mg}$ of mass (at $0 \mathrm{~V}$ ), a $10 \mu \mathrm{N}$ load is applied on the platform. The simulation result of the complete model under a load of 10 $\mu \mathrm{N}$ is shown in Figure 2-13. The stiffness of the platform-connected moving fingers is not sufficient; they bend excessively under the load condition. As a result, the platform and the connected beam move down $19 \mu \mathrm{m}$ from their initial positions (Figure 2-13). The initial gap distance between the moving fingers and the fixed fingers is $2 \mu \mathrm{m}$ as defined by the PolyMUMPS design rules. At this displacement, the platform and the moving fingers will be wedged underneath the fixed fingers and the substrate. As a result, an out-of-plane motion cannot be produced. Therefore, some modifications are required in the design to improve the stiffness of the platform-connected moving finger.

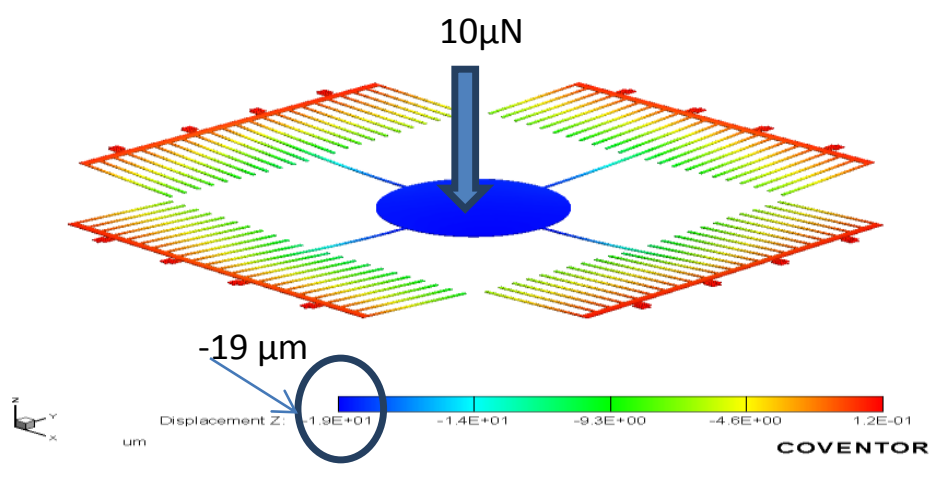

Figure 2-13. Simulation result under load condition. 


\subsubsection{Design of Support Beam}

The stiffness of the platform-connected moving finger can be improved by increasing its width. However, an increase in the width of the platform-connected moving finger will reduce the number of fingers; ultimately the output force will be decreased. Another option is to add a support beam. A support beam is added by connecting all moving fingers. All individual forces, developed in a single finger will be transferred to the support beam. From the center of the support beam, a new beam called the connecting beam is used to connect the platform and the support beam. The connecting beam does not bend because of its short length and large width. The simulation results of a single actuator with the support beam and a complete model subject to a $10 \mu \mathrm{N}$ load are shown in Figure 2-14.

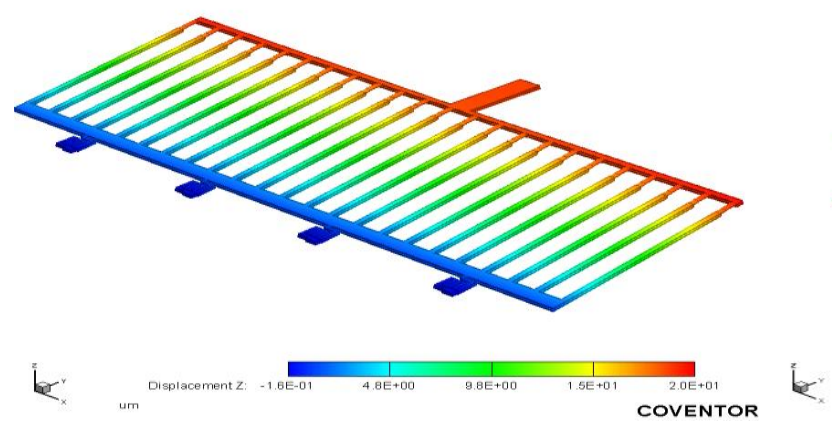

(a)
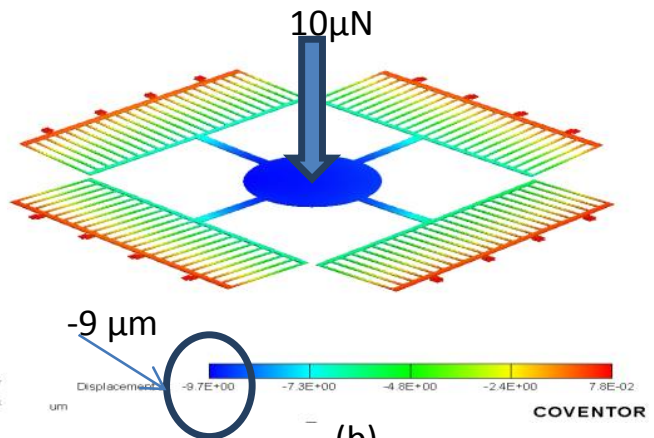

(b)

Figure 2-14. Simulation results (a) of single actuator with supporting beam (b) The Completed model under load $10 \mu \mathrm{N}$.

\subsubsection{Thickness of the MEMS Actuator}

Figure 2-14 shows that by using a support beam, the displacement of the platform in the negative z-direction is decreased from $19 \mu \mathrm{m}$ to $9 \mu \mathrm{m}$. The stiffness of the structure is still insufficient as it would not be able to hold the required mass. The platform should be at the same level under the load condition to produce an out-of-plane displacement. The stiffness of 
the moveable structure is low because the thickness is only $2 \mu \mathrm{m}$. The stiffness of the moveable structure can be improved by increasing the thickness. An iterative approach is used in Coventor to determine a suitable thickness to hold the mass of $1 \mathrm{mg}$. The appropriate thickness was estimated to be $10 \mu \mathrm{m}$. However, due to the restrictions of the commercial fabrication processes, such as PolyMUMPs, only a $3.5 \mu \mathrm{m}$ thickness can be achieved with polysilicon material. Simulation results of the model at $10 \mathrm{um}$ and $3.5 \mathrm{um}$ thickness under loading conditions are shown in Figure 2-15.
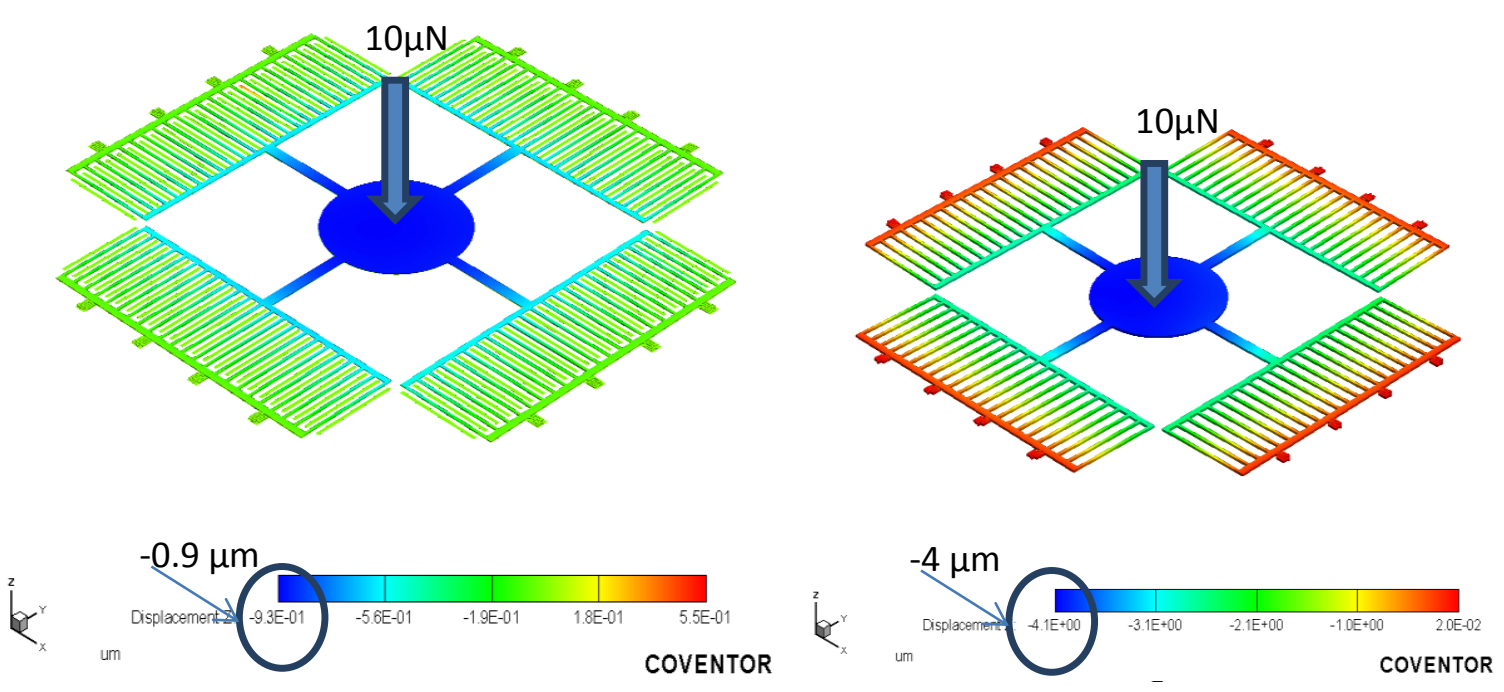

Figure 2-15. Simulation results when subject to a load of $10 \mu N$ (a) $10 \mu \mathrm{m}$ thickness (b) $3.5 \mu \mathrm{m}$ thickness.

\subsubsection{Holding Platform with Electrostatic Springs}

A thickness of $3.5 \mu \mathrm{m}$ was not suitable to hold the platform at its initial position under the loading condition. Further additional support was required to hold the platform at the initial level against the load. For this purpose, large serpentine rotational springs were added to hold the platform at the initial level. An iterative approach was used in Coventor software to design 
springs for an optimal stiffness value. These springs provide extra stiffness to hold the platform when subject to the load.

In order to improve the performance, the mechanical springs are also integrated with aligned and unaligned fixed fingers such that they would work on the MEMS repulsive principle. Given an input voltage, electrostatic springs can provide out-of-plane stroke to support the actuator for out-of-plane motion. Figure 2-16 shows a 3D model of the electrostatic springs (width: $650 \mu \mathrm{m}$, length: $1000 \mu \mathrm{m}$ ) and simulation results of the model with these springs:

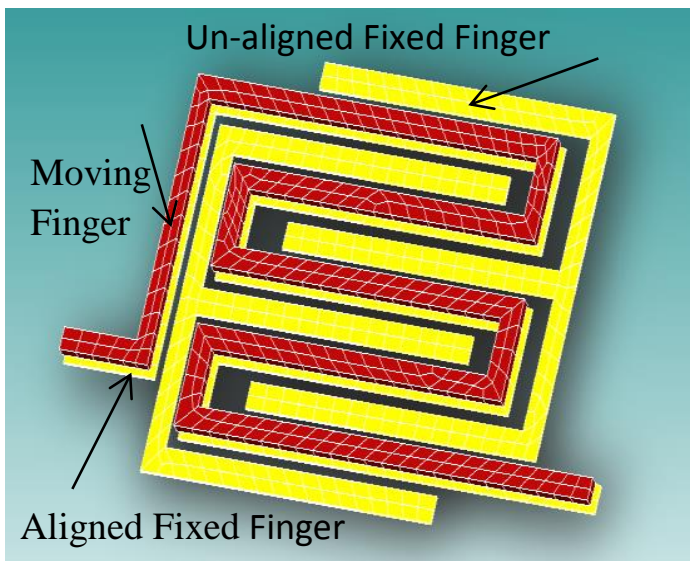

(a)
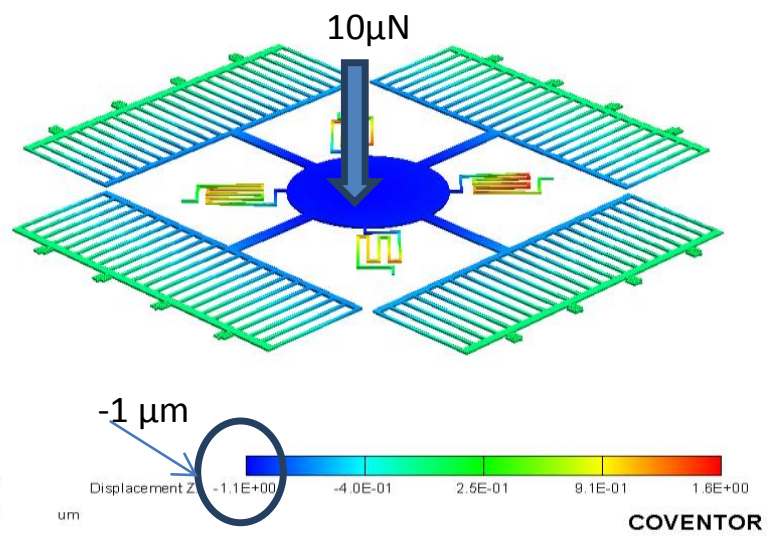

(b)

Figure 2-16. Electrostatic springs (a) 3D model (b) Simulation result with payload platform.

Results show that when the electrostatic springs are excited, the loaded platform will move from $-4 \mu \mathrm{m}$ to $-1 \mu \mathrm{m}$. This distance value is adequate in order to keep the moveable structure away from the substrate.

\subsection{Simulation Results of MEMS Actuators with Payload}

A final completed model in 2D AutoCAD is shown in Appendix A. The complete model was simulated at $100 \mathrm{~V}$. The out-of-plane displacement and angular rotation were found to be $31 \mu \mathrm{m}$ 
and $0.3^{\circ}$, respectively (shown in Figure 2-17), while the out-of-plane force was $40 \mu \mathrm{N}$. The first modal frequency of the proposed structure was also estimated and was found to be $313 \mathrm{~Hz}$.
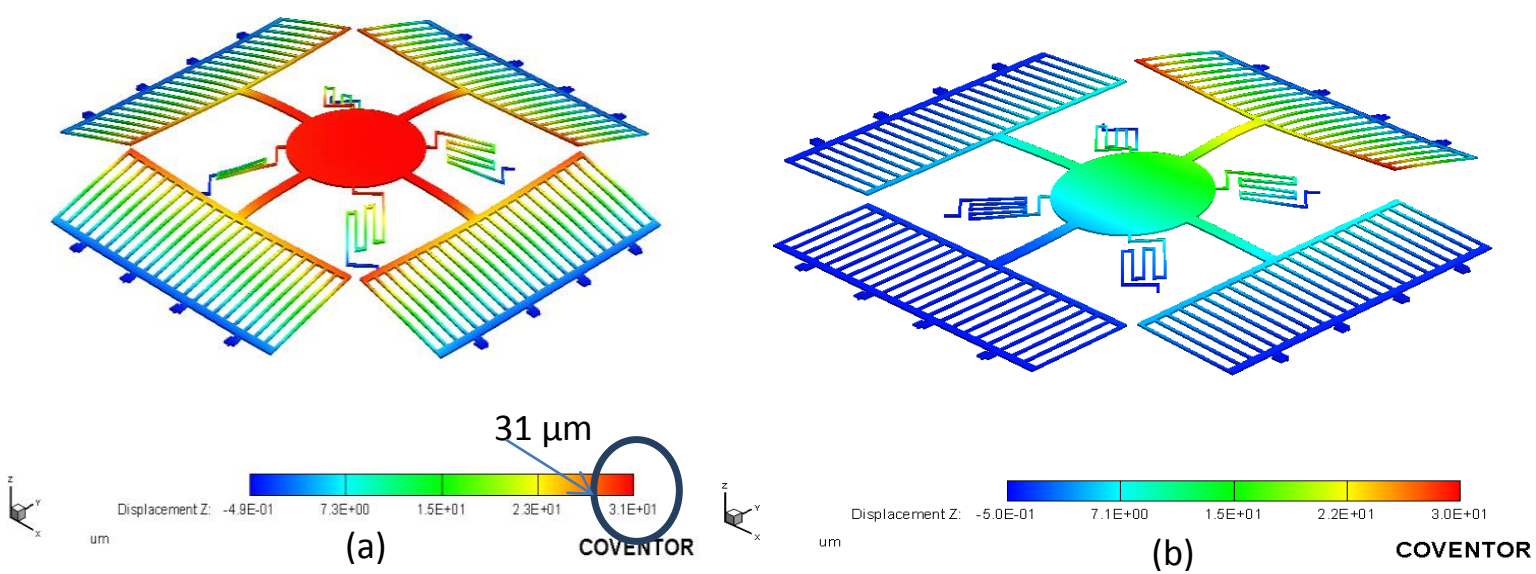

Figure 2-17. Simulation results at $100 \mathrm{~V}$ (a) translation displacement (b) angular rotation when only one actuator is actuated.

\subsubsection{Simulation Results of MEMS Actuators under the Load Condition}

A disk of mass of $1 \mathrm{mg}$ was attached on the platform and the gravitational acceleration was applied to measure the static and dynamic responses of the actuator. When $100 \mathrm{~V}$ is applied, the out-of plane displacement and angular rotation were found to be $12 \mu \mathrm{m}$ and $0.2^{\circ}$ degree, respectively. Simulation results are shown in Figure 2-18.
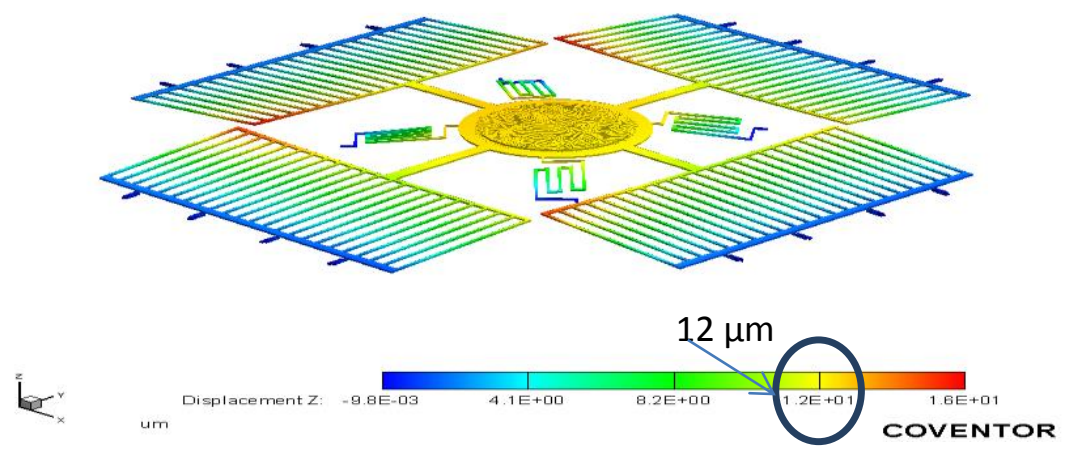

Figure 2-18. Payload platform under a loading condition. 
Stress Analysis under a load of $1 \mathrm{mg}$ (equivalent to $10 \mu \mathrm{N}$ ) shows that the maximum stress value is $33 \mathrm{MPa}$. The first modal frequency of the structure at $1 \mathrm{mg}$ mass load is computed to be $185 \mathrm{~Hz}$.

\subsection{Chapter Summary}

In this chapter, an existing analytical model was used to optimize two important design parameters, finger width and moving finger length to produce a large out-of-plane force. Linear and rotational stiffnesses of the anchor springs were computed. Simulation results, independent of mesh size, of a single actuator show $9.5 \mu \mathrm{N}$ and $35 \mu \mathrm{m}$ out-of-plane force and displacement, respectively, at $75 \mathrm{~V}$. A complete model with the platform was developed using four actuators to hold or lift a mass of $1 \mathrm{mg}$. A variety of different techniques, such as supported beam, increment in the thickness, and electrostatic springs were used to increase the stiffness of the model. The optimum design of the platform was determined using Coventor software, through an iterative approach. The thickness of the complete model was increased from $2 \mu \mathrm{m}$ to $3.5 \mu \mathrm{m}$ and the payload platform was connected with electrostatic rotational springs. A simulated model of the MEMS repulsive actuator with a payload platform shows an out-of-plane displacement of

$31 \mu \mathrm{m}$ without load, and $12 \mu \mathrm{m}$ with load conditions, while the out-of-plane force is $40 \mu \mathrm{N}$ at $100 \mathrm{~V}$. 


\section{Chapter 3}

\section{Prototype Fabrication}

This chapter reviews the PolyMUMPs process for developing a double thickness MEMS repulsive actuator with a holding platform. Development of a supported beam and a shield layer under the constraints of design rules is also discussed.

A commercial PolyMUMPs process allows three polysilicon layers (Poly0, Poly1 and Poly2) and one metal layer to develop the structure. These layers are deposited on the insulated silicon substrate based on the design rules [37]. Sacrificial oxide layers between each polysilicon layer are deposited to support the structure. Finally, all the oxide layers are etched by a $49 \% \mathrm{HF}$ solution, and the structure is released. Small holes are used across the polysilicon layers (Poly1 and Poly2) and metal to make sure that the $49 \%$ HF solution is accessible to the oxide layers under the polysilicon structure. Dimples are also used to avoid potential problems with stiction.

\subsection{Double-Layer (Poly1+Poly2) Actuator}

The proposed actuator was developed using PolyMUMPs. The mask layers Poly0, Poly1 and Poly2 were used to build the fixed and moveable part of the actuator. A side view of double thickness MEMS repulsive actuator with fixed and moving fingers is shown in Figure 3-1.

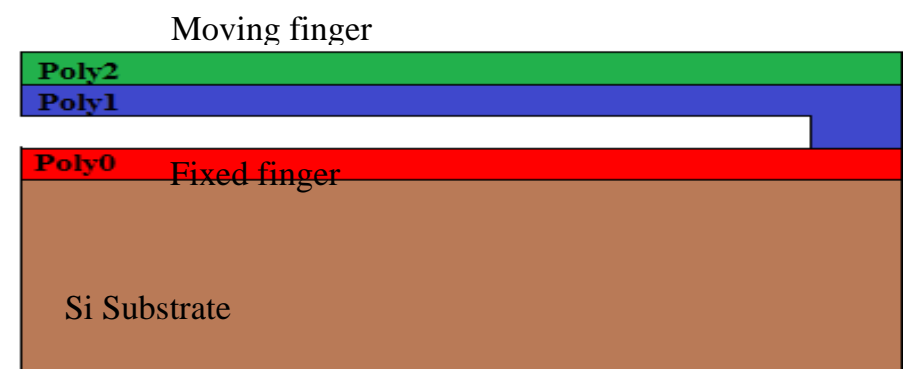

Figure 3-1 Side view of a double-layer actuator 
A step by step procedure to develop the double thickness MEMS repulsive actuator in PolyMUMPs is shown in Appendix B.

The design rule specifies that Poly2 should be enclosed by Poly1 and that Poly1 should be enclosed by Poly0 with a minimum enclosure of $4 \mu \mathrm{m}$. The thickness of the actuator was increased using an etching mask layer Poly1_Poly2_Via to connect Poly2 and Poly1. Due to design modifications, the finger widths of Poly1 and Poly2 layers were reduced to $52 \mu \mathrm{m}$ and 44 $\mu \mathrm{m}$, respectively, compared to the finger width of the Poly0 layer, which is $60 \mu \mathrm{m}$. The reduction in width will affect the performance of the actuator in comparison to the analytical and simulation model. The width of the fixed and moving fingers with respect to their layers is shown in Figure 3-2

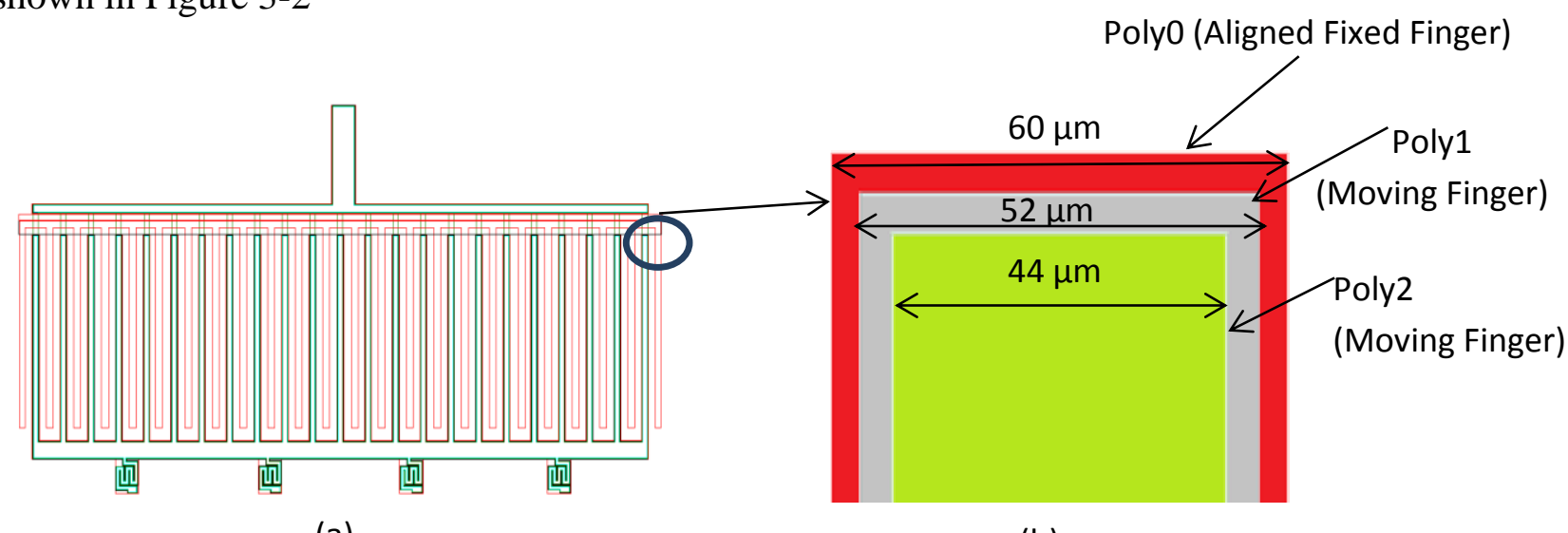

(a)

(b)

Figure 3-2. Top view of (a) Single actuator (b) Exploded top view of fingertip showing structural layers.

For double thickness actuator, the minimum feature size is limited. The minimum width $\left(w_{a}\right)$ of an anchor spring for two turns $(\mathrm{N}=2)$ is $34 \mu \mathrm{m}$ to facilitate Poly1, Poly2 and Poly1_Poly2_Via. The designing of an anchor spring based on the design rule is shown in Figure 3-3. 


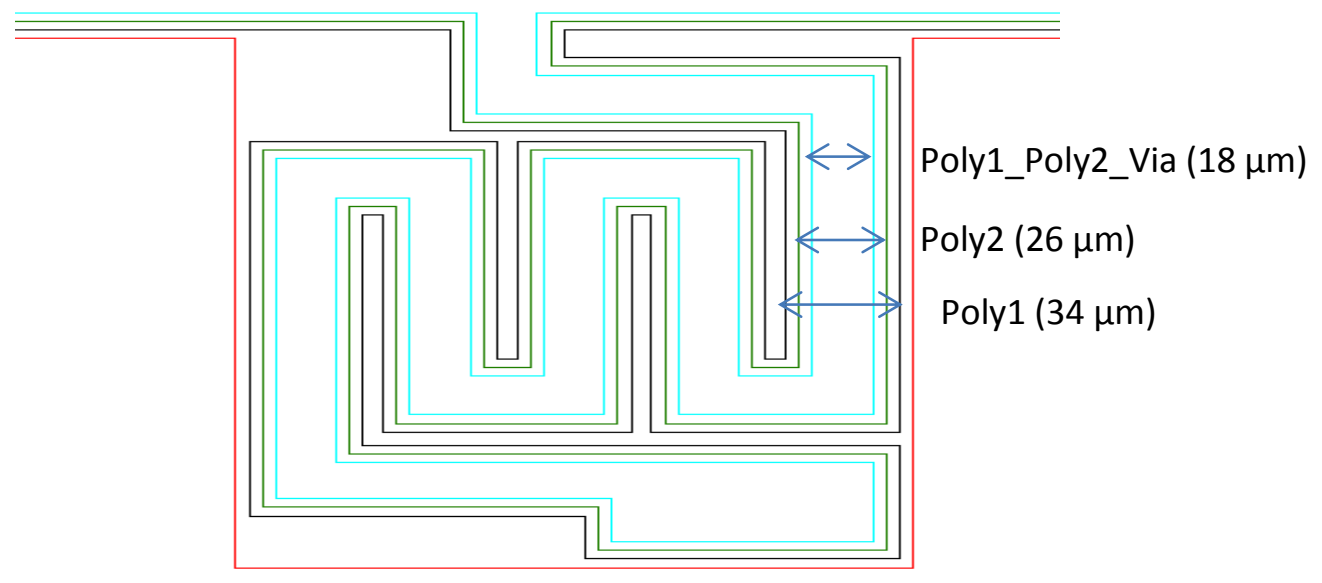

Figure 3-3. Width of each of the poly layers for the anchor springs.

\subsection{Designing of a Supported Beam with Shielded Layer}

As discussed in the previous chapter, a support beam of moving fingers is required to hold the platform when subject to the load condition. Designing a support beam in accordance with the design rules of PolyMUMPs was a significant challenge. In the development of a support beam, it was found that the moving fingers overlap the connecting beam of the unaligned fixed fingers. The moving fingers and the connecting beam of the unaligned fixed fingers have opposite electric potential polarities. As a result, an attractive force will be developed between the moving fingers and the connecting beam. Since the attractive force is greater than the repulsive force, the moving fingers will be attracted by the connecting beam, so an out-of-plane displacement will not be achieved.

According to Faraday's Law, the electrostatic field effect of a charge-carrying object can be cancelled if the object is enclosed by another object of a similar material with no charge value (i.e., neutral) [38]. The enclosed object is called a shield. An extra polysilicon layer was required to create a shield between the moving fingers and the connected beam of the unaligned 
fingers. As the PolyMUMPs process is limited to use only three polysilicon layers, which were already used in building the double-thickness actuator, no additional polysilicon layer was available for developing a shield.

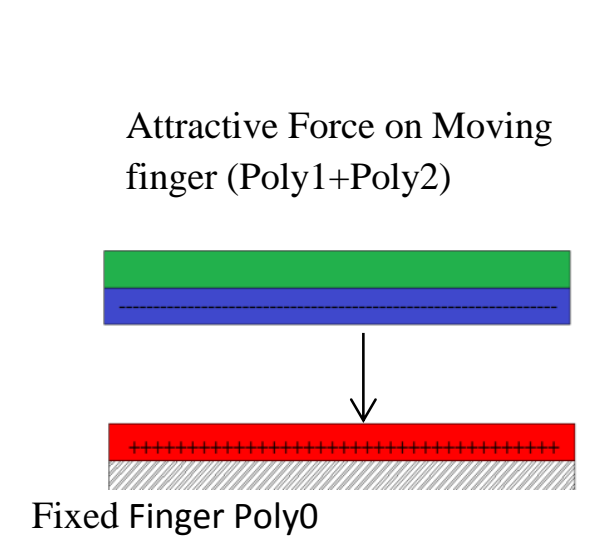

(a)

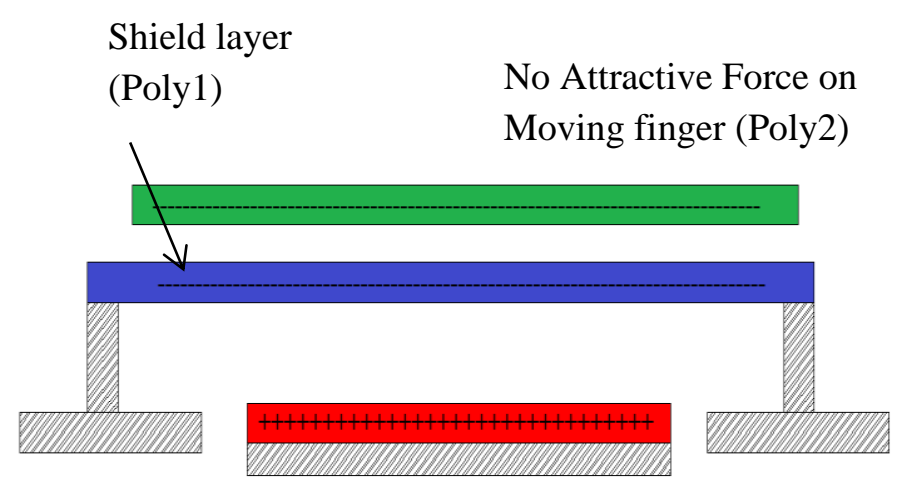

(b)

Figure 3-4. Shield-layer effect (a) Attractive force between fixed and moving finger (b) No attractive force between fixed and moving finger (Poly2) under the shield layer of Poly1.

To address this problem, where the moving fingers have double thickness, some portion of the Poly1 layer was removed and used to make a suspended bridge over the region of the connected beam. This portion of the Poly1 layer is called a shield layer. Figure 3-4 shows the effect of the shield over the connecting beam of a fixed finger. This shield layer cannot completely enclose the connecting beam. But using a shield layer of a larger width than the connecting beam will provide the necessary shielding. The width of the shield layer is $150 \mu \mathrm{m}$ while the width of the connecting beam is only $50 \mu \mathrm{m}$. Anchors were used to suspend the shield layer over the desired region.

Removing a portion of the Poly1 from the moving finger may affect the performance of the actuator. However, the portion with reduced thickness is only $8 \%$ of the total length of $1800 \mu \mathrm{m}$. 2D and 3D views of the multi-fingers set with the arrangement of shield layer, Poly1, Poly2 and Poly0, are shown in Figures 3-5 and 3-6. 


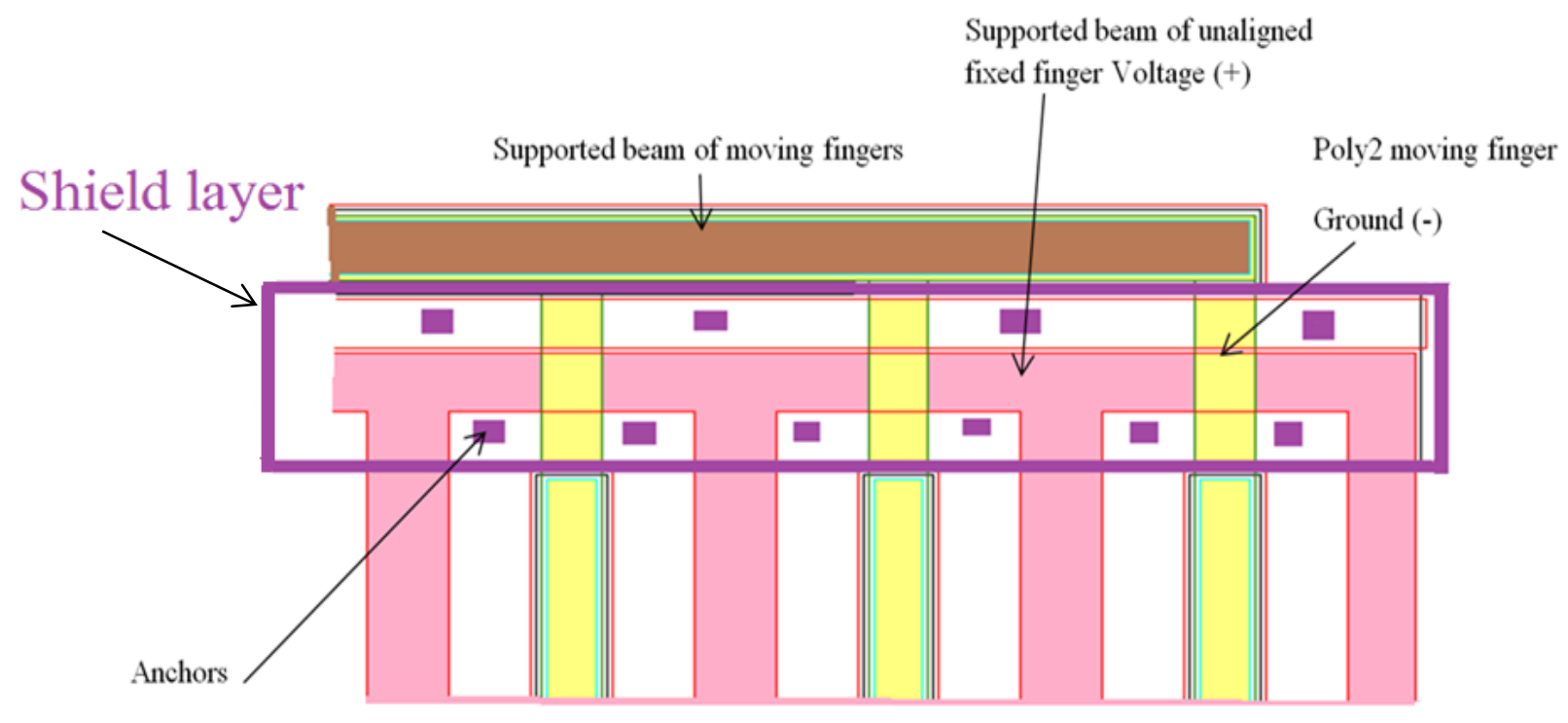

Figure 3-5. Top view of moving and fixed fingers with shielded layer.

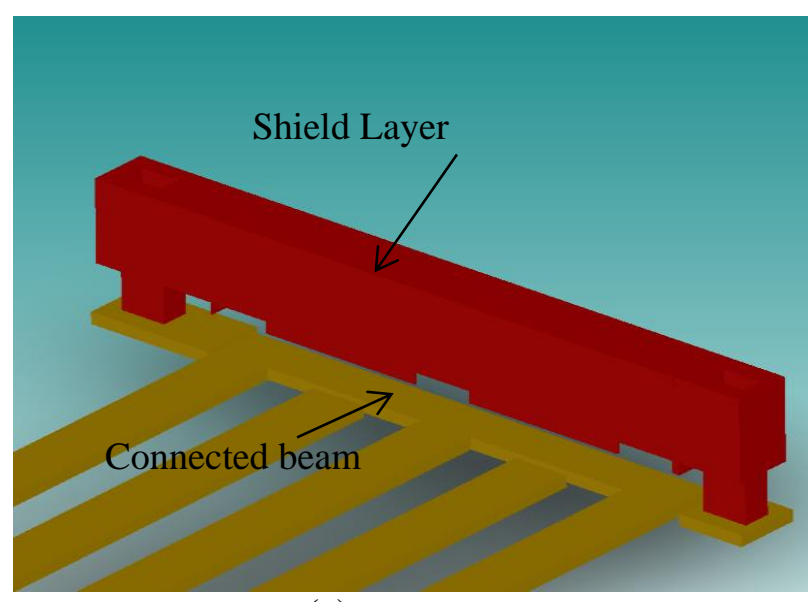

(a)

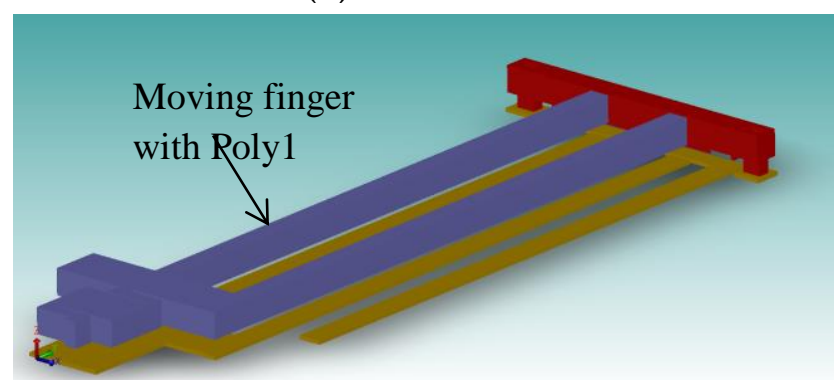

(c)

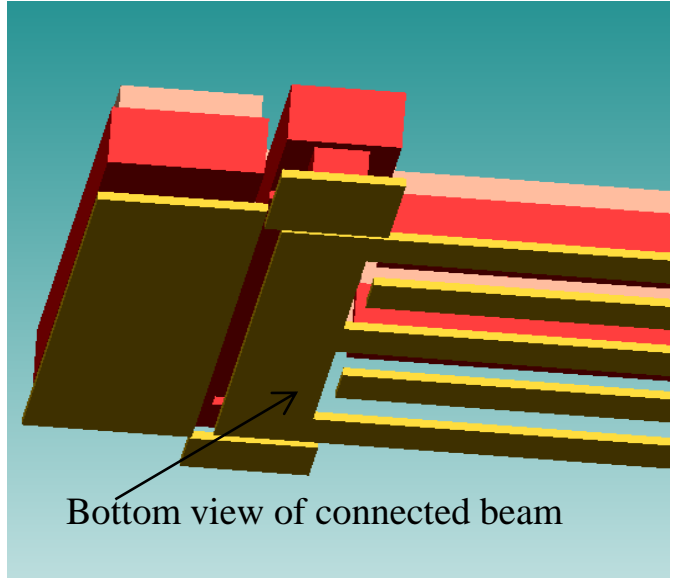

(b)

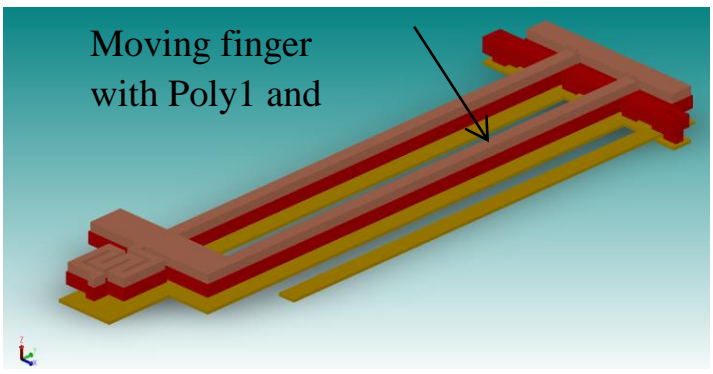

(d)

Figure 3-6. 3D view of a one-unit cell moving and fixed fingers with shielded layer (a) shield layer on connecting beam (b) Bottom view of connecting beam and shield layer (c) Moving finger Poly1 and shield layer Poly1 (d) Moving finger with Poly2 and Poly1. 
After developing the desired structure in the PolyMUMPs process, the structure will be released and packaged. Packaging CPGA 84 was used to package the chip for conducting the experiment on the prototype. The bonding diagram of the prototype with CPGA84 is shown in subsection A-2 of Appendix A.

\subsection{Chapter Summary}

A double thickness MEMS repulsive actuator was developed using Poly0, Poly1 and Poly2 silicon layers. The width of the moving fingers and the anchor springs were adjusted with respect to the design rules. A shield layer comprised of Poly1 was developed to prevent the attractive force between the fixed and the moving fingers. 


\section{Chapter 4}

\section{Experimental Analysis}

This chapter reviews the experimental analysis performed on the fabricated prototype. The analysis includes determining static and dynamic characteristics of the prototype. Static and dynamic measurements were performed using a Zygo profilometer and a position sensor diode. Effectiveness of the shield's layer, and preventive action to avoid the short circuit during the experiment, are also discussed.

Three variants of prototype chips were developed based on their applications: packaged, released, and un-released. The packaged chip is developed using CPGA 84 (through hole pin) packaging and it is used to determine the static and dynamic characteristics of the prototype. The released chip can be used to test the initial condition of the chip, before applying the packaging. The un-released chips are developed for a load test. A mass should be attached on the chip in un-released condition in order to protect the chip from any damages.

The prototype is shown in Figure 4-1. Before applying the voltage, the electrical characteristics of the chip are measured to make sure the electrostatic field can be generated for the desired tasks. The initial resistance and capacitance of the actuator is measured through a capacitance meter (Agilent 4288A) as shown in Table 4-1.

Table 4-1. Initial electrical measurements of the chip before applying voltages.

\begin{tabular}{|c|c|c|c|c|}
\hline Actuators & $\begin{array}{c}\text { Series capacitance } \\
\mathrm{pF}( \pm 0.1 \mathrm{pF})\end{array}$ & $\begin{array}{c}\text { Parallel capacitance } \\
\mathrm{pF}( \pm 0.1 \mathrm{pF})\end{array}$ & $\begin{array}{c}\text { Parallel resistance } \\
\mathrm{M} \Omega( \pm 0.001 \mathrm{M} \Omega)\end{array}$ & $\begin{array}{c}\text { Series resistance } \\
\mathrm{K} \Omega( \pm 0.01 \mathrm{~K} \Omega)\end{array}$ \\
\hline South & 294 & 294 & 65 & 4.5 \\
\hline East & 294 & 294 & 65 & 4.5 \\
\hline North & 294 & 294 & 65 & 4.4 \\
\hline West & 294 & 294 & 65 & 4.5 \\
\hline
\end{tabular}




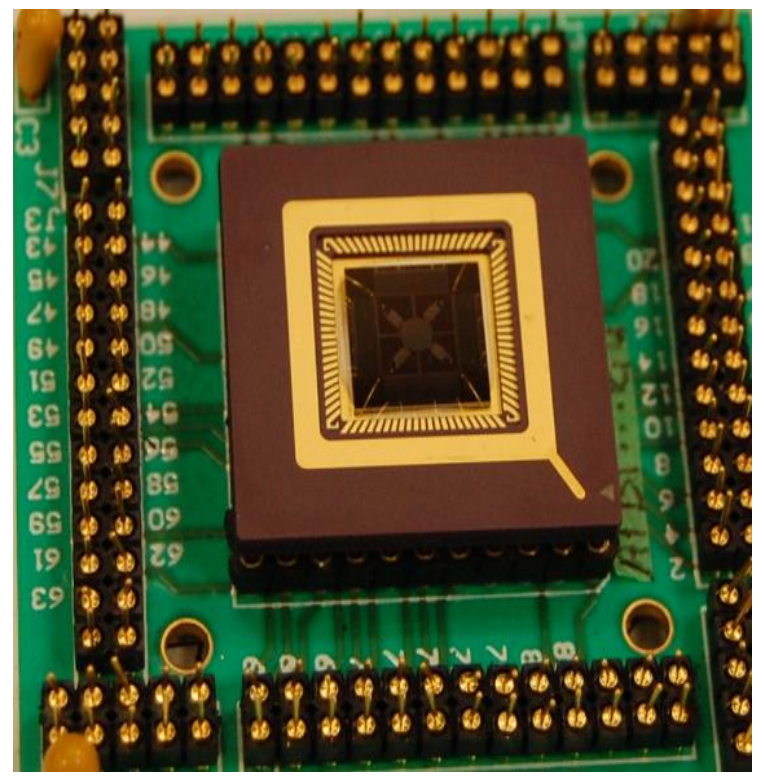

(a)

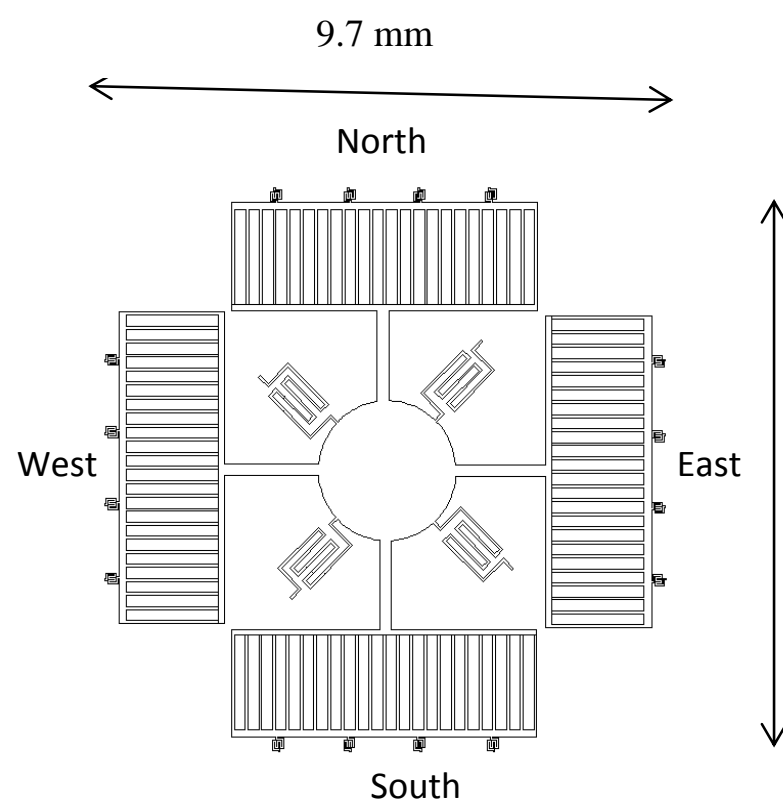

(b)

Figure 4-1. Prototype (a) packaged chip (b) Top view of the chip in 2D drawing.

\subsection{Experimental Setup}

The electrical connections were built for testing the chip in a voltage range of $0 \mathrm{~V} \sim 160 \mathrm{~V}$. A two channels output arbitrary function generator (Tektronix DP03014) was used to provide a DC or a sinusoidal input of $0 \mathrm{~V} \sim 5 \mathrm{~V}$. An amplifier (Tegam 2350) of constant gain was used to amplify the input voltage up to $160 \mathrm{~V}$. An oscilloscope (Tektronix AFG 3102) with sampling frequency of $100 \mathrm{KHz}$ was used to measure the static and dynamic responses of the chip. The setup details of each test are mentioned in their respective sections. 


\subsection{Static Measurements}

\subsubsection{Translational measurements}

A 3D optical profiler (Zygo New View 6300) was used to measure the static translational displacement. Initially, the chip was inspected at $0 \mathrm{~V}$ under the Zygo profilometer. The setup is shown in Figure 4-2.

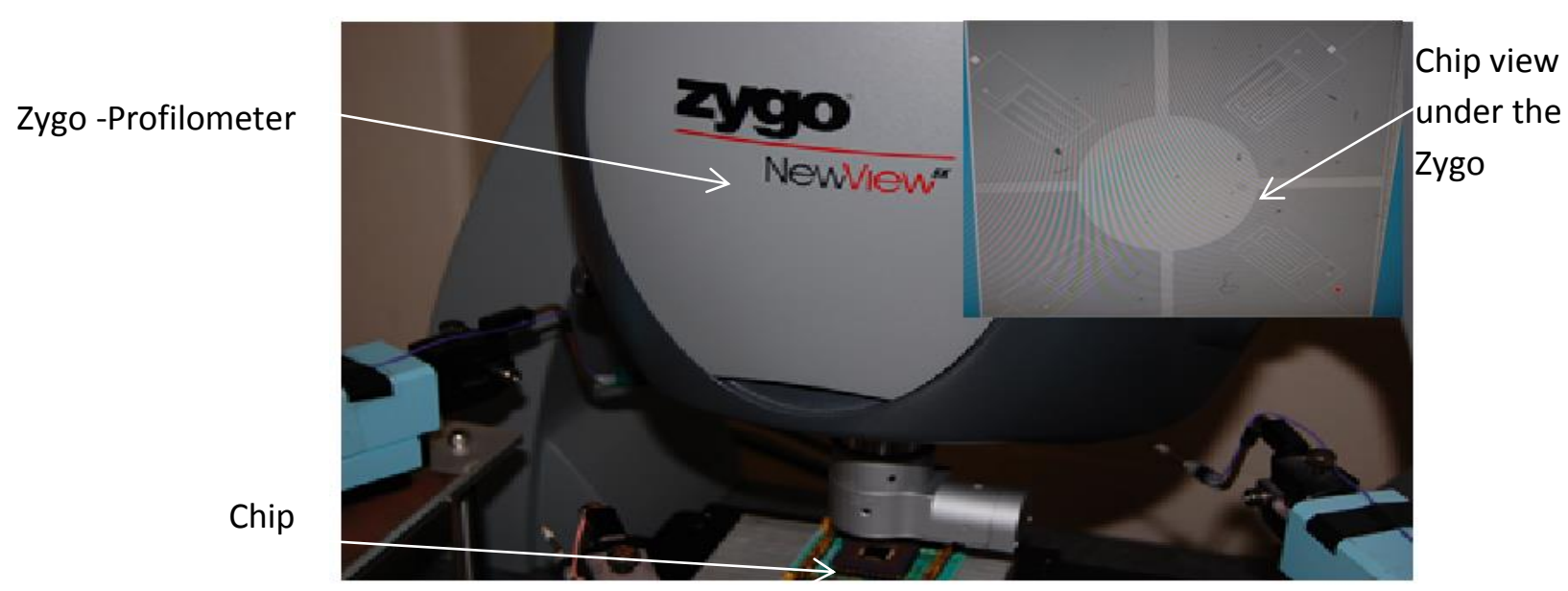

Figure 4-2. Chip inspected under the Zygo profilometer.

At $0 \mathrm{~V}$, it is found that the platform was elevated $15 \mu \mathrm{m} \pm 0.1 \mu \mathrm{m}$ from the substrate. Measurements were taken across the cross-section $\mathrm{AB}$ as shown in Figure 4-3. However, at $0 \mathrm{~V}$ the distance between the substrate and the platform should be $5.5 \mu \mathrm{m}$ (thickness of the platform $3.5 \mu \mathrm{m}$ and $2 \mu \mathrm{m}$ initial gaps distance from the substrate). The one possible reason of the initial elevation of the platform from the substrate is the releasing stress. When the chip was released, the moveable part of the actuator went through the experience of the releasing stress. In the current design the platform is directly connected with the rigid beam, therefore, once the platform is released, it lifts up in order to compensate for the stress. 
Out-of-plane displacement was observed by inducing the same voltage into all four actuators ranging from $0 \mathrm{~V}$ to $120 \mathrm{~V}$. The maximum out-of-plane displacement is found to be $15 \mu \mathrm{m} \pm 0.1$ $\mu \mathrm{m}$ across $\mathrm{AB}$ from the initial position as shown in Figure 4-3. The relation between the applied voltage and the out-of-plane displacement is also shown in Figure 4-4.
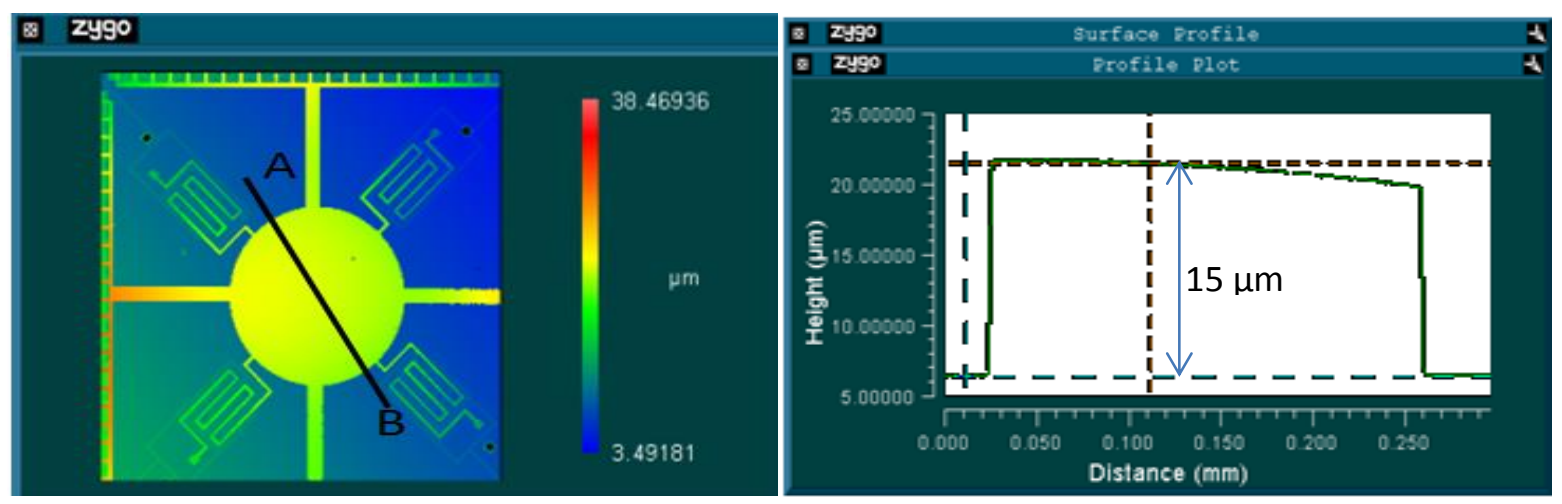

(a)
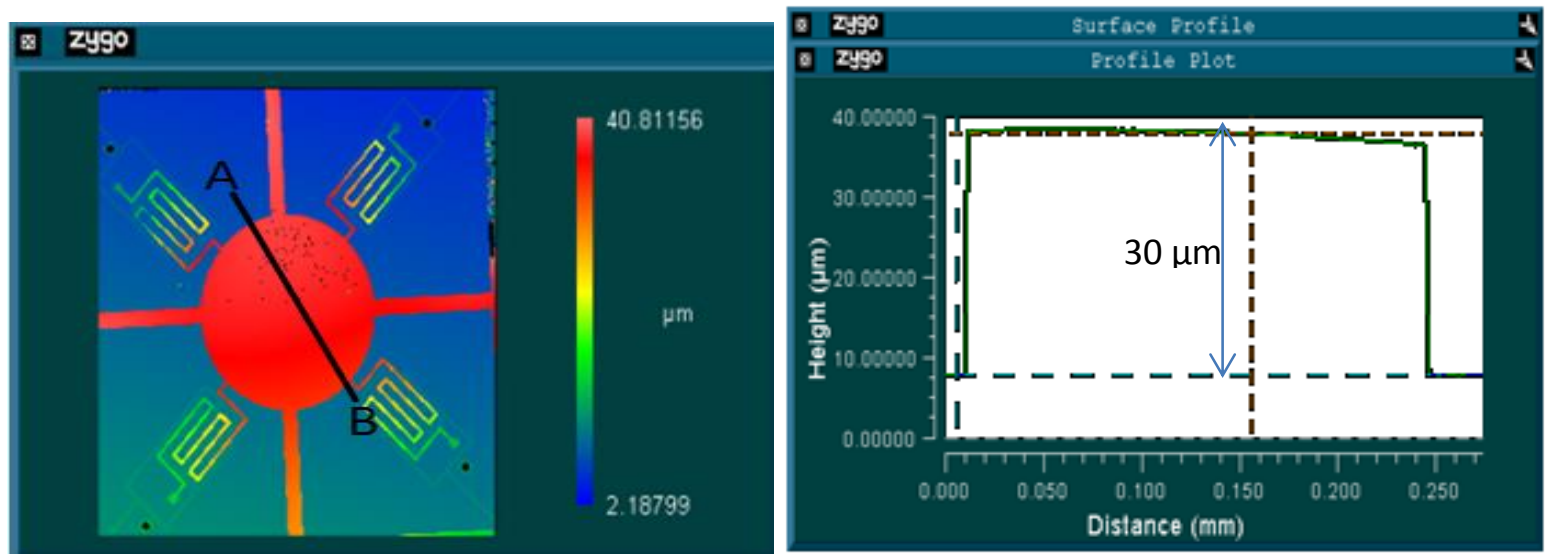

(b)

Figure 4-3. 2D views and corresponding surface profile across $A B$ under the Zygo Profilometer (a) At 0 V (b) At 120 


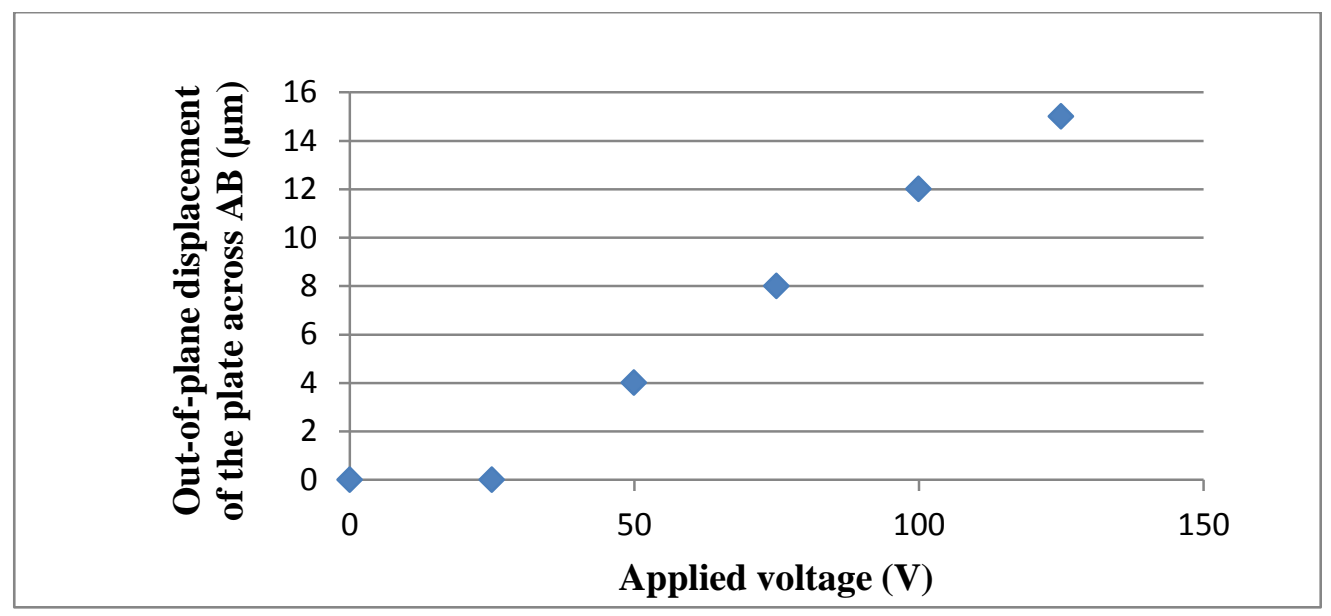

Figure 4-4. Out-of-plane displacement of the platform $0 \mathrm{~V} \sim 120 \mathrm{~V}$.

The simulation results show in Figure (2-16) that an out-of-plane displacement at $100 \mathrm{~V}$ is 30 $\mu \mathrm{m}$. One of the reasons in difference of static measurements is the result of the initial elevation of the platform from the substrate. Due to the initial elevation, the electrostatic force was reduced in comparison to that of the simulation model and caused short stroke length. It can be assumed that if the platform was not elevated at $0 \mathrm{~V}$, the translation displacement could be reached up to $25 \mu \mathrm{m}$ at $120 \mathrm{~V}$, which is close to the simulation result.

\subsubsection{Rotational Measurements using Position Sensor Diode (PSD)}

The static rotational displacement of the prototype was determined by bouncing a well collimated laser beam off the platform surface. As the platform is rotated, the angular displacement can be calculated by measuring the location of the reflected laser spot using a PSD, setup is shown in Figure 4-5. The alignment of a laser light is critical for measuring angular displacement precisely. Before the prototype is actuated, PSD needs to be aligned perpendicular to the laser beam that reflected from the platform. The beam reflected from the PSD should also be collinear with the entering beam, as shown in the 3D model setup (Figure 4- 
5b). The position of the laser spot on the PSD can be measured using an oscilloscope: a displacement of $1 \mu \mathrm{m}$ on the PSD gives an output of $1 \mathrm{mV}$. The $\mathrm{x}-\mathrm{y}$ position of the laser dot changes as the platform moves. The platform can be rotated about the $\mathrm{x}$ and $\mathrm{y}$ axes by applying a voltage to each actuator independently. The rotation of the platform about the $\mathrm{x}$ and $\mathrm{y}$ axes can be achieved by actuating the north/south and east/west actuators, respectively. The distance $\mathrm{D}$ between the PSD and the prototype is fixed, and the displacement of the laser spot on the PSD can be determined with the oscilloscope. Thus, the angular displacement can be computed using simple trigonometry. Figure 4-6 shows angular displacement of the platform as function of the voltage applied to the actuators.

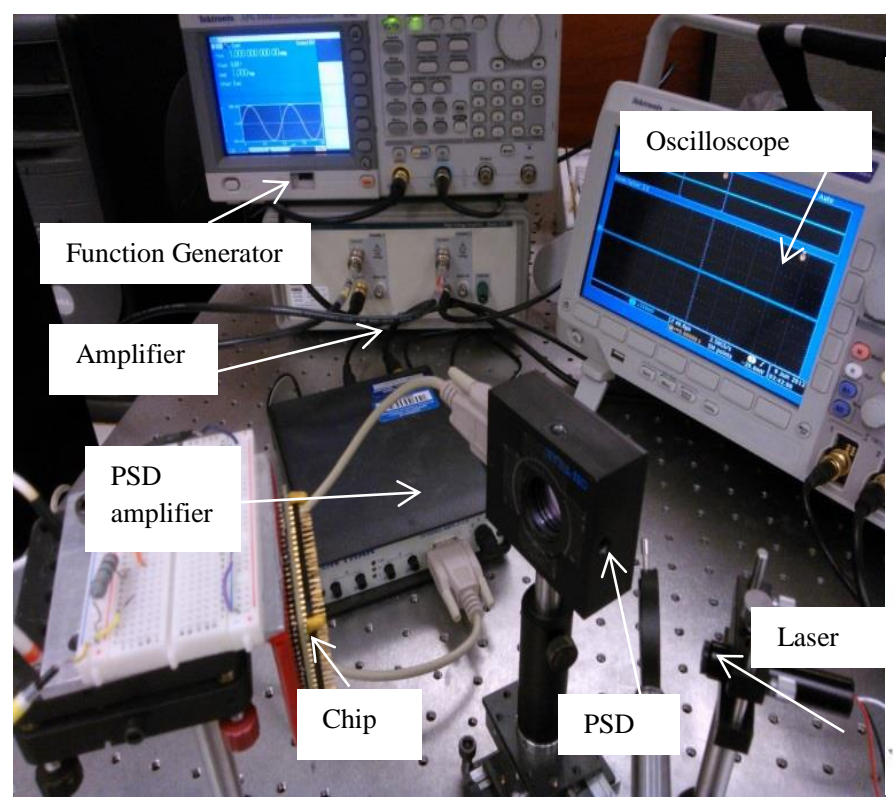

(a)

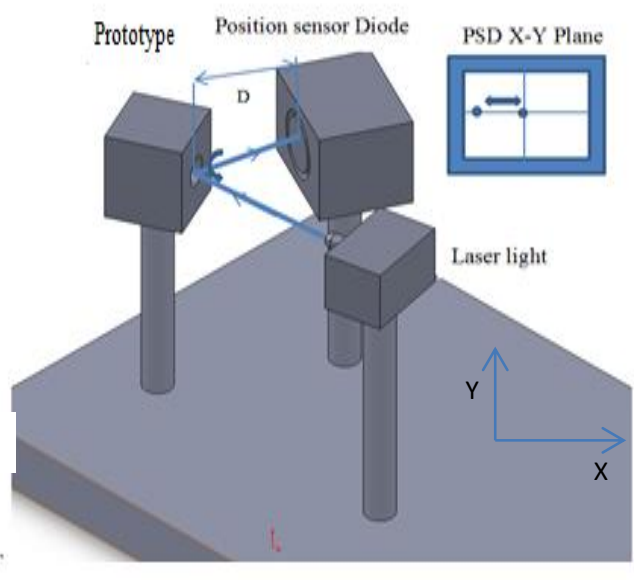

(b)

Figure 4-5. Angular displacement measurements (a) Experimental setup (b) 3D view of PSD setup with laser alignment. 


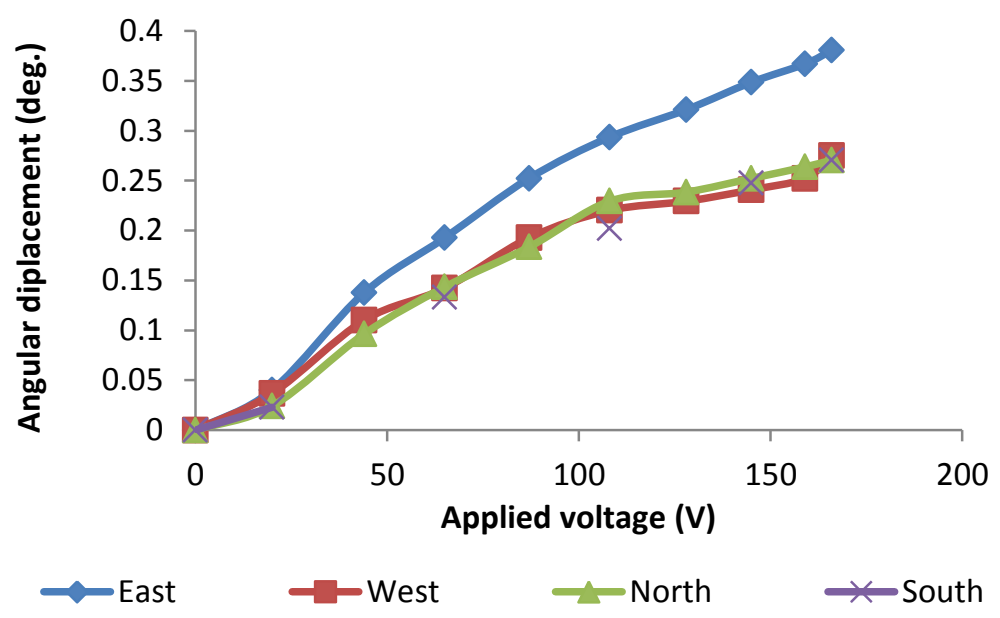

(a)

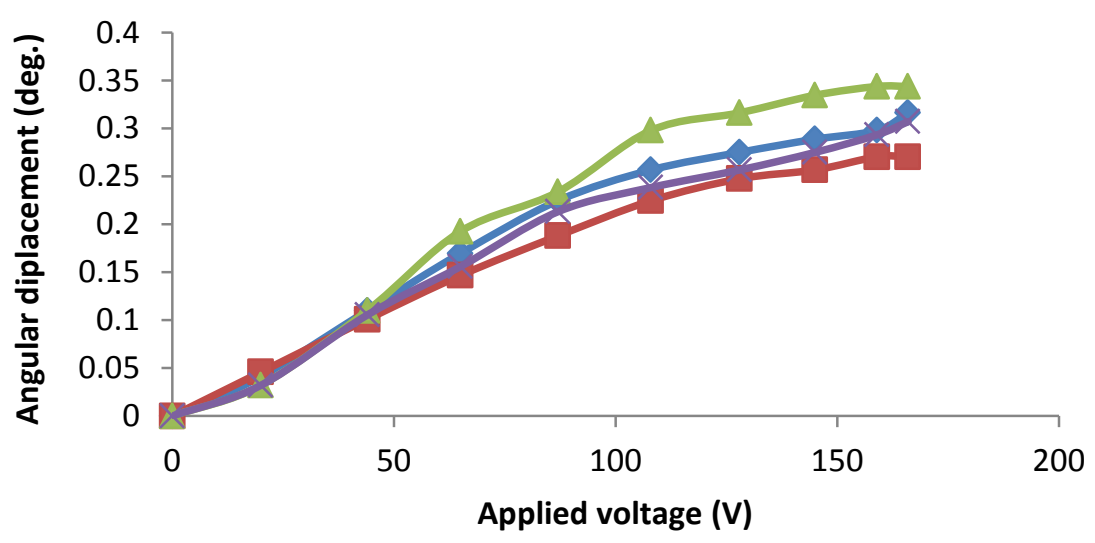

(b)

Figure 4-6. Angular displacements based on the displacement of the laser spot in (a) $\mathrm{X}$ direction (b) $\mathrm{Y}$ direction.

If the platform rotates either the x-axis or y-axis, the bouncing spot of the laser should move in only one direction at a time: either the x-direction or the y-direction. In this case, since the bouncing spot moves in both directions, it can be inferred that the rotational axis of the platform is neither the $\mathrm{x}$-axis nor the $\mathrm{y}$-axis, but lies between them due to rigid connections between the platform and the actuators. The platform is directly connected to the actuators by the beams. As a result, a stroke introduced by one actuator affects other actuators through the platform, so a 
combined motion (translation and rotation) is observed in the $x-y$ plane. Since the current setup is limited to determining the rotation only about the $\mathrm{x}$-axis or the $\mathrm{y}$-axis, and cannot be used to detect rotation about other axes, another method will be required to measure the angular displacement of the platform.

\subsubsection{Rotational Measurements under the Zygo Profilometer}

The angular displacement of the platform can be measured precisely under the Zygo profilometer. Under the Zygo profilometer, rotation can be measured between the x-axis and yaxis of the platform, which was not possible using the PSD. A voltage $0 \mathrm{~V} \sim 120 \mathrm{~V}$ was applied to each actuator at a time, in order to measure the angular displacement of the platform. The outof-plane displacement between the upper and lower portions of the platform was measured about the rotational axis. The diameter of the platform is known, so the angular displacement can be computed using simple trigonometry.

When only the west actuator was activated, the north-west portion of the platform moved up to $3.5 \mu \mathrm{m}$ from the central axis AA of the platform and the opposite side east-south portion of the platform moves down to $3 \mu \mathrm{m}$ from the same axis. Therefore, a total displacement of $6.5 \mu \mathrm{m}$ is achieved between the two portions of the platform which is equivalent to rotating the platform to $0.188^{\circ}$ about the axis AA. Similar rotations were observed in east and north actuators about their axis of rotations. Results are shown in Figure 4-7.

The relation between the applied voltage, ranging from $0 \mathrm{~V} \sim 160 \mathrm{~V}$ and the angular displacement of the platform, is also shown in Figure 4-8. 


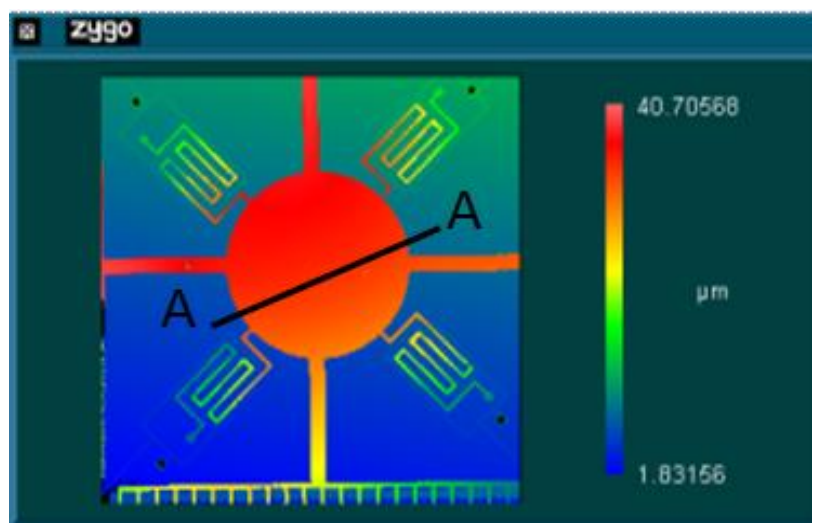

(a)

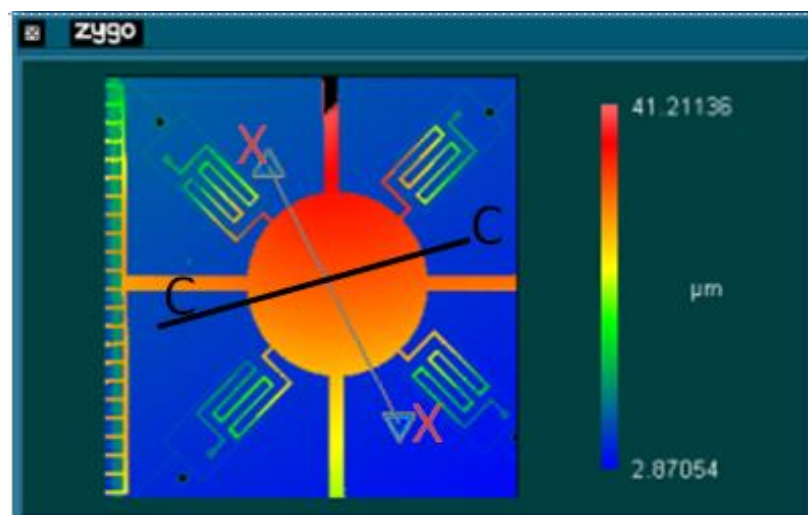

(c)

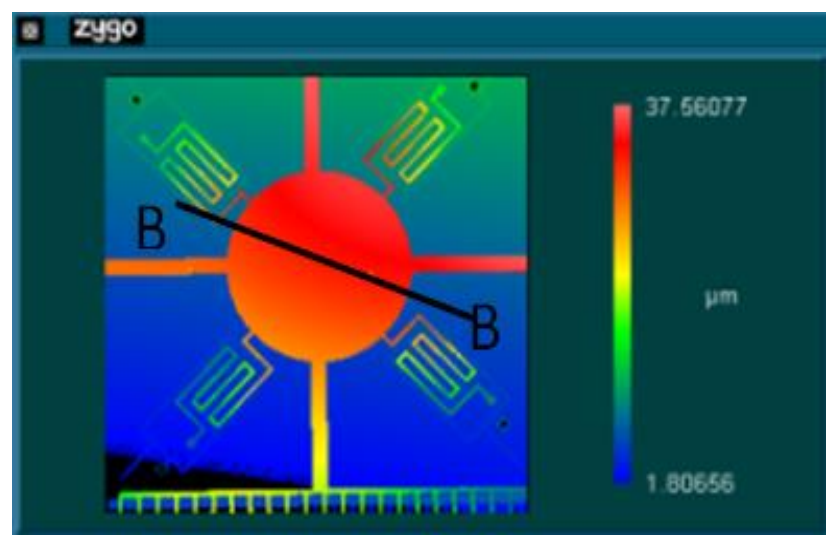

(b)

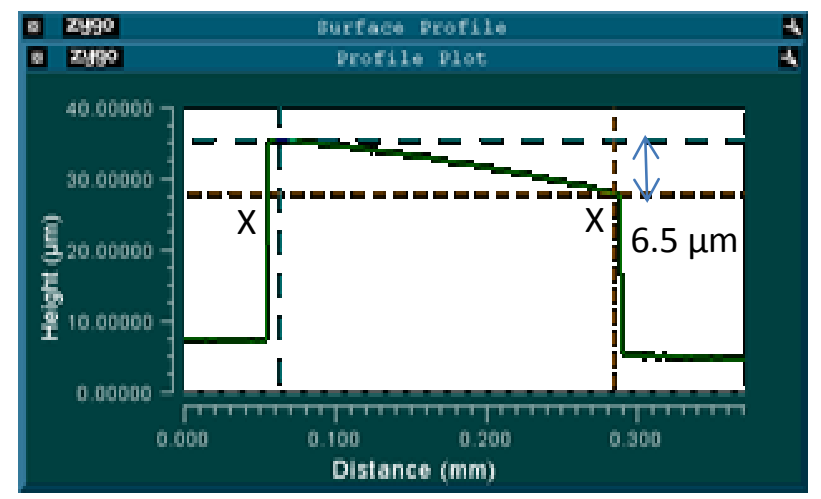

(d)

Figure 4-7. Rotation of the platform in 2D view under the Zygo Profilometer, when $120 \mathrm{~V}$ is applied on (a) West actuator (b) East actuator (c) North actuator (d) Surface profile along XX, shows the out-of-plane displacement of the north actuator about $\mathrm{CC}$ axis.

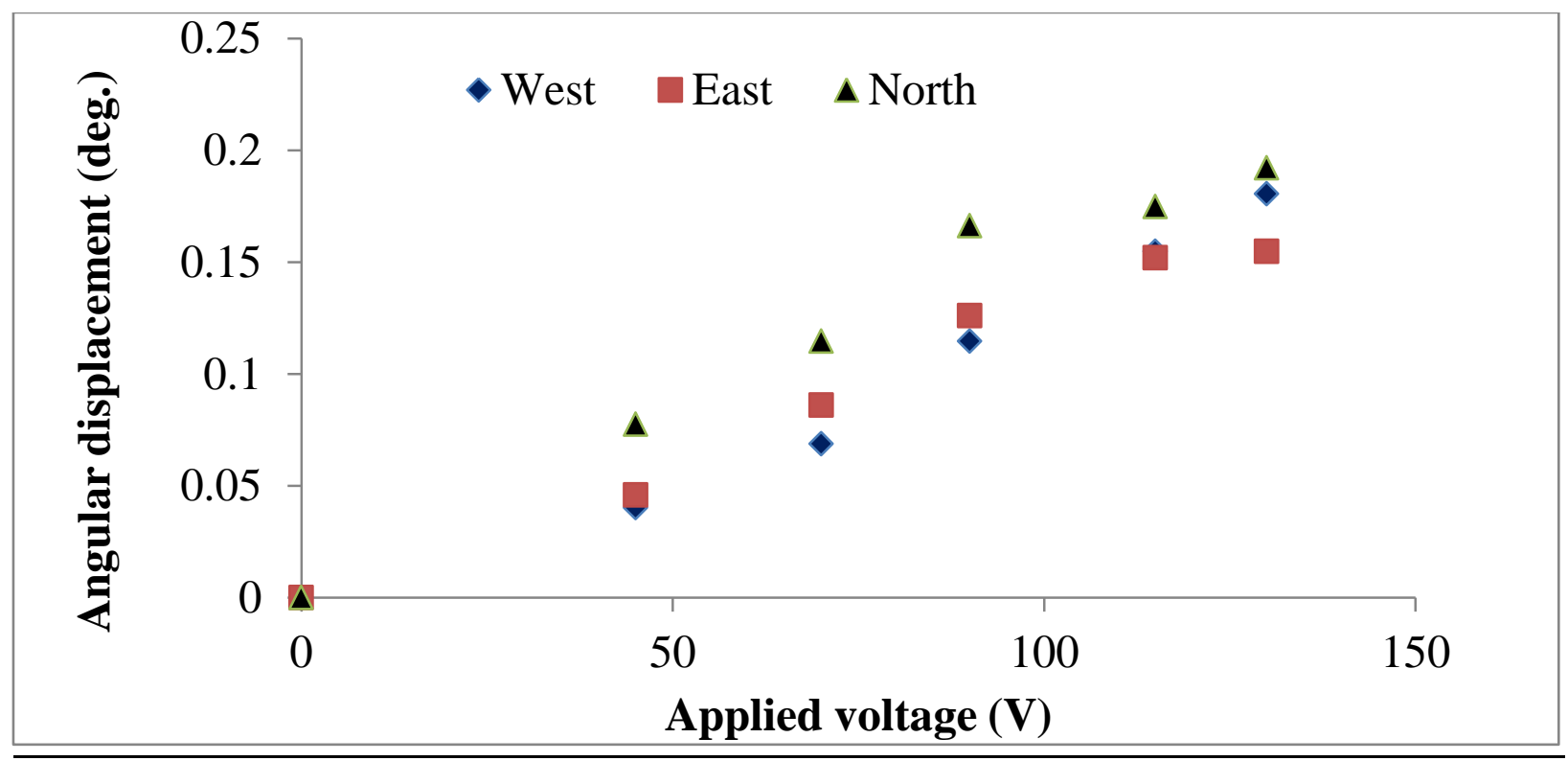

Figure 4-8. Angular rotation of the platform due to each actuator under the Zygo Profilometer. 


\subsection{Dynamic Measurements}

The dynamics measurements were performed using a PSD, with a similar setup as discussed in section 4.2.2. Two types of dynamic responses, time response and frequency response, were measured.

\subsubsection{Time Response}

The time response of the platform in rotational motion was examined by observing the performance of the platform as a step input voltage varying from $0 \mathrm{~V} \sim 160 \mathrm{~V}$. Experimental results (shown in Table 4-2) indicate that the average rise time and the average fall time are $14.5 \mathrm{~ms}$ and $3625 \mathrm{~ms}(3.6 \mathrm{sec})$, respectively. The system response is over damped in both directions.

Table 4-2. Average rise and fall time for prototype.

\begin{tabular}{|l|c|c|}
\hline \multirow{2}{*}{ Actuator } & \multicolumn{2}{|c|}{ Time Responses (ms) } \\
\cline { 2 - 3 } & Rise Time & Fall Time \\
\hline North & 10 & 3000 \\
\hline South & 16 & 3500 \\
\hline East & 13 & 4000 \\
\hline West & 19 & 4000 \\
\hline Average & 14.5 & 3625 \\
\hline
\end{tabular}

The time response was measured at a $100 \mathrm{KHz}$ sampling frequency. The data points $(100,000)$ were saved against each response in order to reconstruct the response for further analysis. The rise time and fall time responses are shown in Figure 4-9. 

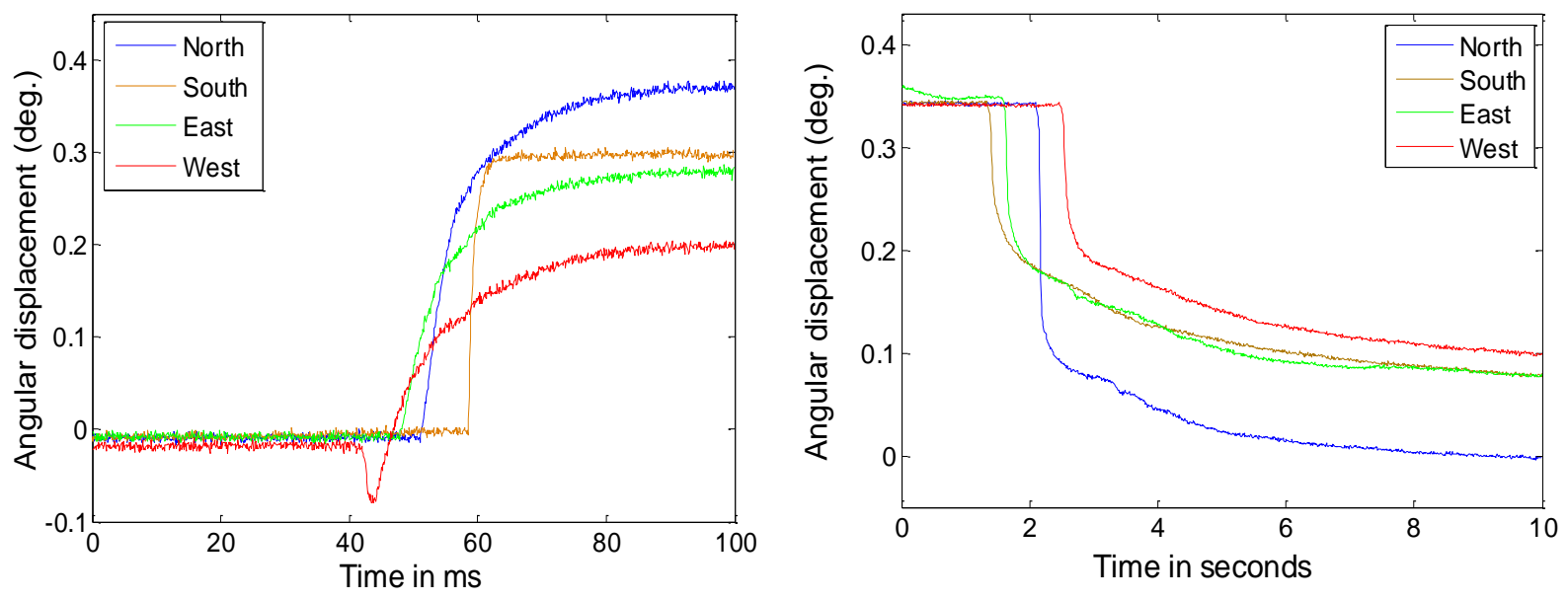

Figure 4-9. Time responses $0 \mathrm{~V} \sim 160 \mathrm{~V}$ (a) Rise time in milliseconds (b) Fall time in seconds.

(a)

(b)

Typically, the fall time is greater than the rise time because of the squeeze film damping effect which increases the fall time by a few milliseconds. Since the size of actuator is larger in comparison to previous MEMS repulsive actuator, the effect of squeeze film damping effect might be significant. In order to investigate the squeeze film damping effect, the chip needs to be packaged under the vacuum condition. Under the vacuum packaging, the rise and fall time are similar because the absence of squeeze film damping effect [39].

Another possible reason for large fall time is might be the discharge behaviour of the capacitors. As discussed in Chapter 1, its operation is governed by simple electrostatic forces. When a voltage is applied, the actuator is charged quickly, producing an electrostatic repulsive force and generating an out-of-plane stroke. When the voltage stimulus is removed, the charge on the actuator dissipates gradually, so the actuator falls slowly. It can also be seen in Figure 49 that nearly $70 \%$ of the downward motion occurs in the first $50 \mathrm{~ms}$, while the remaining $30 \%$ takes $3.575 \mathrm{sec}$. This shows that when the actuators are detached from the supply voltage, the electrostatic field is immediately reduced, but it does not vanish completely. Roughly, 10\% - 
$20 \%$ of the electrostatic field remains providing resistance to the downward motion until the field vanishes completely.

To extend the investigation, a voltage was applied and removed from all the actuators at same time to observe the rise time and the fall time responses. Figure 4-10 shows that the rise time decreases to less than $10 \mathrm{~ms}$ and the fall time decreases to $3 \mathrm{sec} ; 85 \%$ of the distance is covered in $50 \mathrm{~ms}$ and the remaining $15 \%$ in $2.95 \mathrm{sec}$. When all the actuators and springs are activated at the same time, they undergo the same motion together, which decreases the rise and fall times.

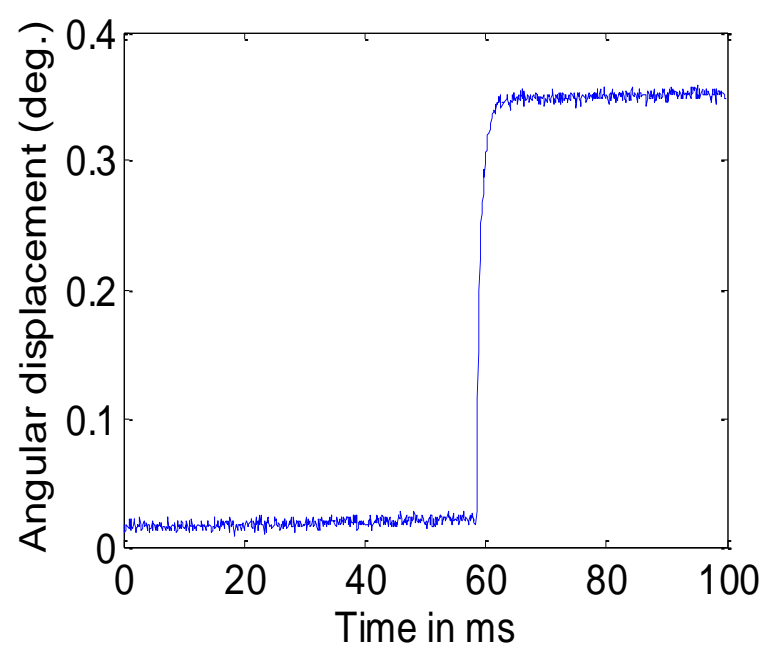

(a)

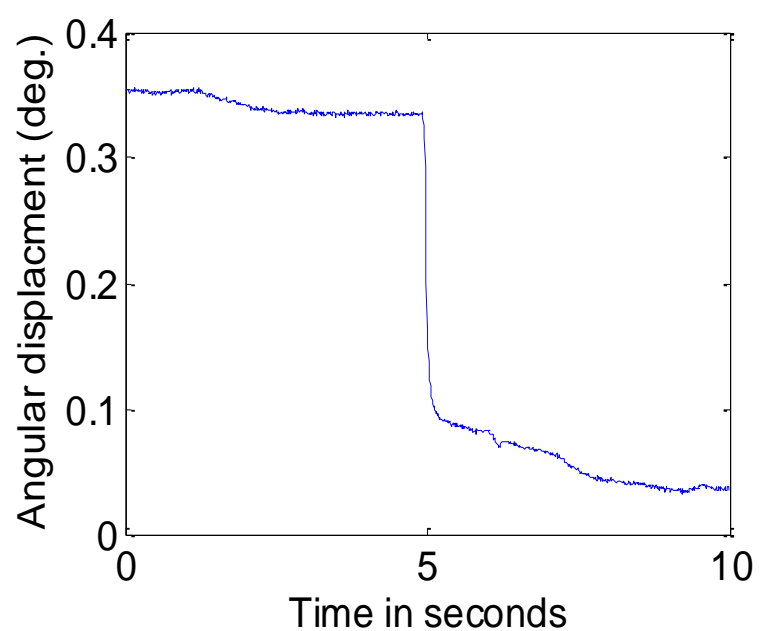

(b)

Figure 4-10. Time responses for all actuators acting together $0 \mathrm{~V} \sim 160 \mathrm{~V}$ (a) Rise time in milliseconds (b) Fall time in seconds.

The effect of the electrostatic field was further analyzed by considering the time response data for a chip that has low resistance. A voltage $(50 \mathrm{~V})$ was applied to the one actuator of the chip and then the time response was observed. The rise and fall time responses are shown in Figure 4-11 and they are very close: rise time $12 \mathrm{~ms}$ and fall time $15 \mathrm{~ms}$, respectively. The electrostatic field is not strong across the fixed and moving fingers in the low resistance chip due to flow of current $(20 \mathrm{~mA})$. Electrostatic field does not provide any resistance to downward motion. 
Therefore, it may suggest that the major reason for large fall time is the effect of electrostatic field. Further analysis will be required to conclude the result.

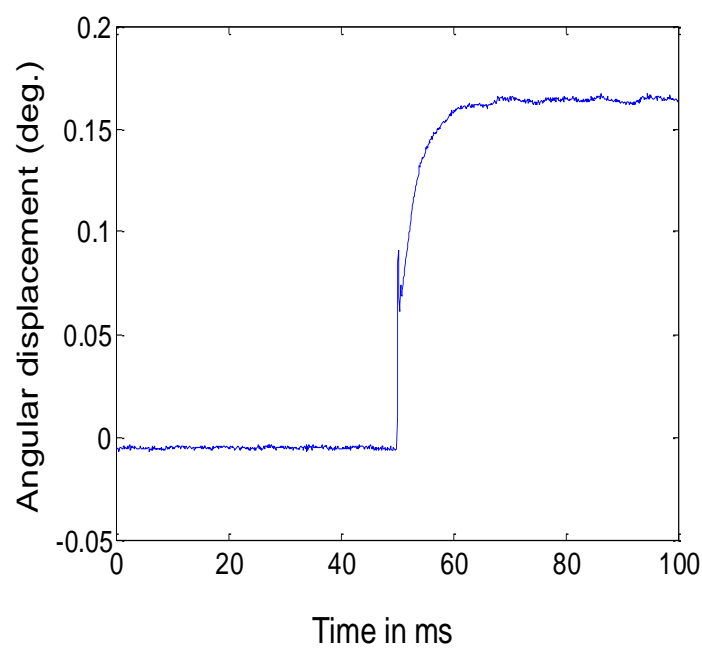

(a)

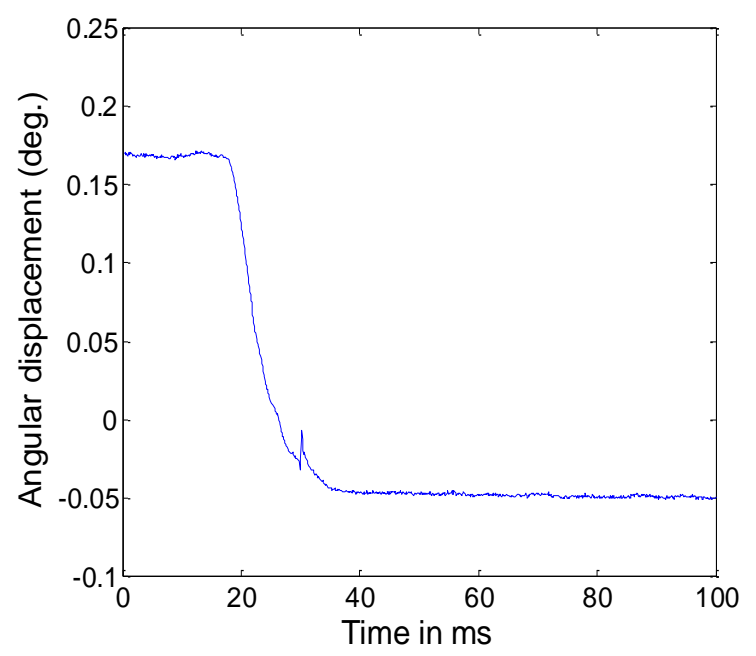

(b)

Figure 4-11. Time responses $0 \mathrm{~V} \sim 160 \mathrm{~V}$ (a) Rise time in milliseconds (b) Fall time in seconds.

\subsubsection{Frequency Response}

The frequency response of the prototype is estimated using the time response. A Fourier transform is applied to the rise time and fall time data to estimate the resonance frequency. The approximate resonance frequency of the prototype is $120 \mathrm{~Hz}$ under the voltage of $0 \mathrm{~V} \sim 160 \mathrm{~V}$ as shown in Figure 4-12.

The resonance frequency is not calculated through the experiment in order to avoid any damages in the chip. At resonance frequency, the motion of the platform is expected to increase 5 to 10 times which may damage the other actuators as a result of of rigid connections. 


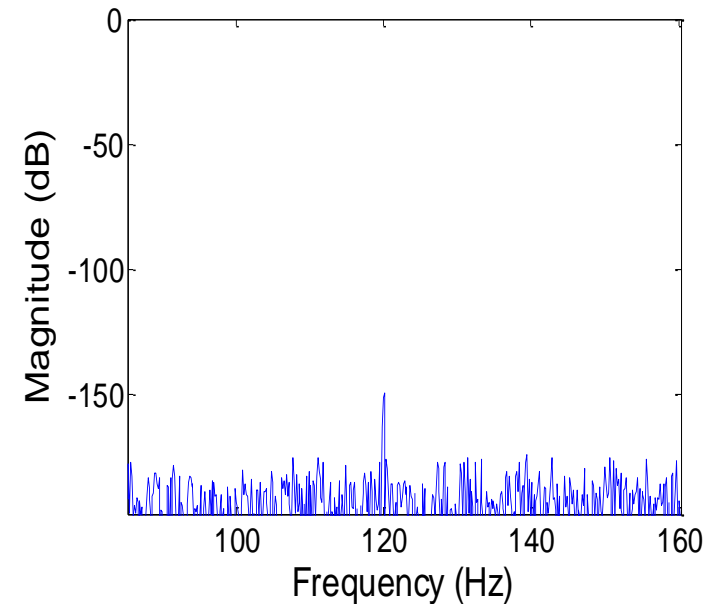

(a)

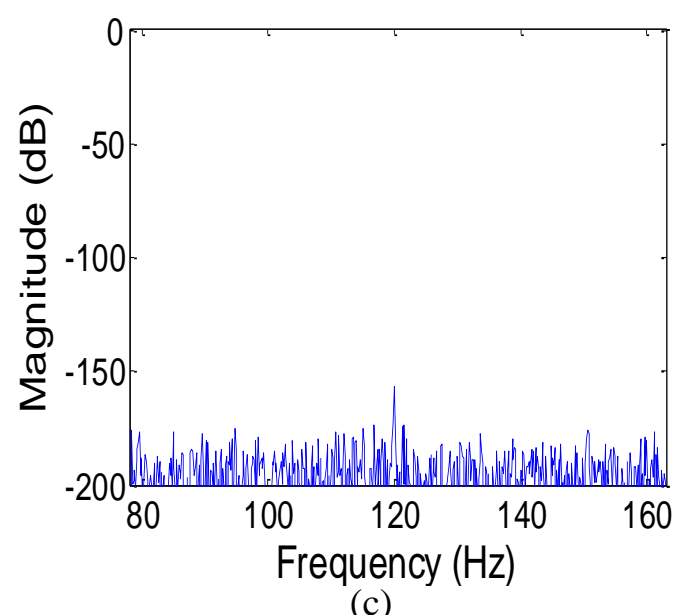

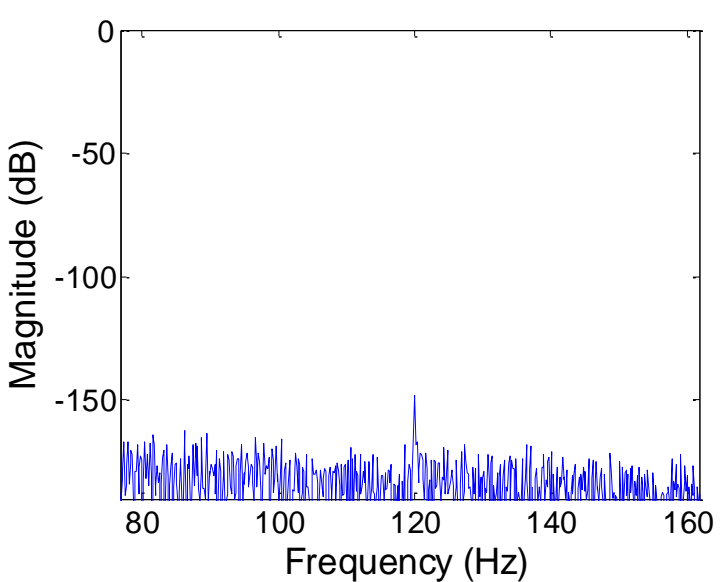

(b)

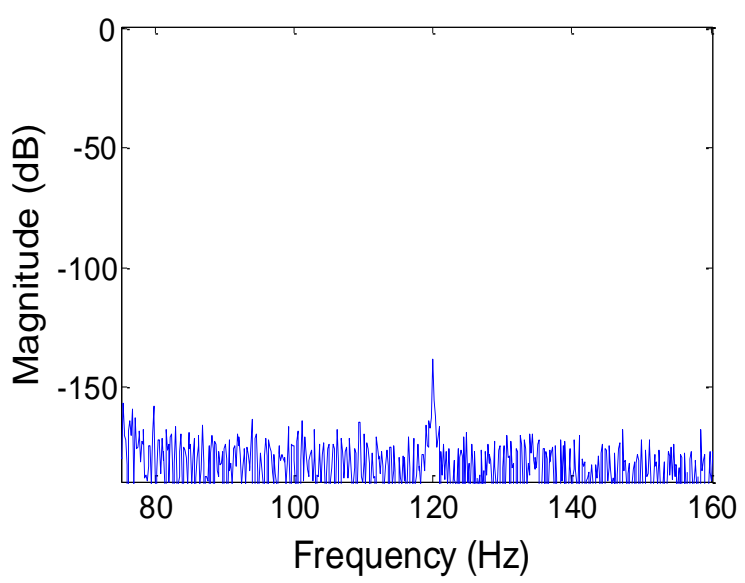

(d)

Figure 4-12. Frequency response under the voltage of $0 \mathrm{~V} \sim 160 \mathrm{~V}$ (a) East actuator (b) West actuator (c) South actuator (d) North actuator .

Figure 4-12 shows that an approximate resonance frequency of each actuator is $120 \mathrm{~Hz} \pm 5 \mathrm{~Hz}$. The amplitudes are not the same for each actuator. The north actuator shows higher amplitude while the south shows lower. The reason for different magnitudes might be because of their different stiffness values. The time response plot (Figure 2-8) also shows that the north actuator travels more distance compared to the south actuator. The stiffness of each actuator might vary with each other because of a double thickness actuator. 
The simulation result shows the first natural frequency is around $300 \mathrm{~Hz}$. The difference between the results is might be the fabrication process of the double-layer actuator. The simulation result is based on a single layer of Poly1 structure with $3.5 \mu \mathrm{m}$ thicknesses. The prototype was built using Poly1 of $2 \mu \mathrm{m}$ thickness and Poly2 of $1.5 \mu \mathrm{m}$ thickness. The etching layer Poly1_Poly2_Via was also used to etch $0.75 \mu \mathrm{m}$ thickness oxide layer between Poly1 and Poly2 in order to connect them. Any deficiency in etching the oxide layer will change the thickness and mass of the actuator, ultimately changing the mechanical properties of the structure. Another reason is might be the different Young's modulus values for polysilicon layers Poly1 and Poly2. The possible values for Young's modulus range from 130 to $188 \mathrm{GPa}$ [40].

\subsection{Testing of Prototype under the Load condition}

So far, the performance of the prototype could not be tested under the load condition because of the time limit. However, procedures for load testing and expected results are described in the following subsections.

\subsubsection{Procedure for Load Test}

The un-released chip will be used for this testing. A mass equivalent to $1 \mathrm{mg}$ will be placed on the payload platform using a micromanipulator. The stress analysis of the structure with $1 \mathrm{mg}$ mass shows the maximum stress value on the platform under the load condition is less than 35 $\mathrm{MPa}$, where the ultimate tensile stress of silicon is $1.20 \mathrm{GPa}$ [30]. Therefore, placement of a 1 mg mass on the platform is safe. 
A material of $1 \mathrm{mg}$ mass should be a solid particle, or a UV curable liquid, but it should not be etchable by $49 \% \mathrm{HF}$ solution. Since the platform has a large number of small holes of size 30 $\mu \mathrm{m}$, using the UV curable liquid is only feasible if it has high viscosity and less curing time.

After placing the mass on the platform, the structure will be released in $49 \%$ HF solution. The released chip will require very careful handling for further processing. By adding the mass on the platform, the natural frequency of the structure will be decreased, and the structure will be more sensitive for handling vibration. The released structure will be enclosed in the packaging to perform the static and dynamic test.

\subsubsection{Expected Experiment Result with Load}

Simulation results (Figure 2-15) show the out-of-plane displacement under the load condition is $12 \mu \mathrm{m}$ at an applied input voltage of $100 \mathrm{~V}$. Coventor software is used to simulate the model with load condition. In Coventor, two different models (MemMech \& MemElectro) are used simultaneously to compute the solution. MemMech deals with the mechanical force due to the load while MemElectro deals with the electrostatic force. Because of two different domains, the response time of each force might not equal. Therefore, the computation with load condition might not be very accurate. Based on the experiment results, the out-of-plane displacement with load might reach $6 \mu \mathrm{m}$ to $8 \mu \mathrm{m}$. However, it is also expected that the initial lifting force from the actuator might increase in comparison to the case without load condition, because initially the platform will move down $1 \mu \mathrm{m}$ to $3 \mu \mathrm{m}$ due to the mass. This will change the initial gap distance between the fixed fingers and moving fingers. Thus, the initial lift force will be increased, ultimately increasing the out-of-plane displacement. Experiments will be required to investigate the characteristics of the chip under the load condition. 


\subsection{Short-Circuit Analysis}

During the initial run of experiments, a short-circuit incident was observed, when the applied voltage exceeded to $50 \mathrm{~V}$. A spark was observed at the junction of the supported beam and platform connected beam. Due to the apparent short-circuit, the resistance of the chip was changed from the initial value, and a flow of high current (10 mA to $70 \mathrm{~mA})$ was observed in different parts of the actuator. Table 4-3 shows chip circuit measurement after the short-circuit.

Table 4-3 Chip circuit measurement after short-circuit.

\begin{tabular}{|l|l|l|l|l|l|}
\hline Actuators & $\begin{array}{l}\text { Parallel } \\
\text { capacitance } \\
\mathrm{pF}\end{array}$ & $\begin{array}{l}\text { Series } \\
\text { Capacitance } \\
\mu \mathrm{F}\end{array}$ & $\begin{array}{l}\text { Series } \\
\text { Resistance } \\
\mathrm{K} \Omega\end{array}$ & $\begin{array}{l}\text { Parallel } \\
\text { Resistance } \\
\mathrm{K} \Omega\end{array}$ & $\begin{array}{l}\text { Current } \\
(\mathrm{mA})\end{array}$ \\
\hline South & 236 & 48.3 & 1.45 & 1.5 & 20 \\
\hline East & 436 & 52 & 1.5 & 1.05 & 30 \\
\hline North & 858 & 110 & 0.500 & 0.52 & 80 \\
\hline West & 147 & 36 & 2.15 & 2.1 & 20 \\
\hline
\end{tabular}

One of the possible reasons of a short-circuit is a limited performance of the shield layer. The shield layer was used to cancel the electrostatic attractive field effect between the moving finger and connected beam. After $50 \mathrm{~V}$, the electrostatic force increases and is not restricted by the shield. As a result, the moving fingers touch the connected beam of unaligned fixed fingers, leading to a short-circuit incident. The resistance of the short-circuit was reduced by three orders of magnitude to the kilo Ohm range. Due to the low resistance, current flows inside the circuit. This high current also has possibility to melt the shield layer and silicon structure.

As per Faraday's law, the shield layer should completely enclose the charge carrying object in order to cancel the electrostatic effect. In the prototype, the shield layer covers only the top area of the connected beam. Since the gap distance between the shield layer and the connected beam 
is only $2 \mu \mathrm{m}$, it was assumed that the shield layer would be able to cancel the electrostatic field effect. This assumption was also validated through the computational analysis.

The discrepancy between the simulation and experimental result regarding the shielding test may be because of software limitation, or its pre-set initial condition (which presupposes that all conductor parts are initially grounded regardless of whether or not they are defined).

In order to avoid the short-circuit, a high resistance of $1 \mathrm{M} \Omega$ was used in series with the electric circuit of the chip. High resistance in series restricts the flow of current to a maximum of $20 \mu \mathrm{A}$ in any situations to guard against a short-circuit. By doing this, the chip was tested up to a high voltage (up to $160 \mathrm{~V}$ ) without observing any incident of a short-circuit. This shows that shortcircuits can be avoided by restricting the flow of current.

\subsection{Chapter Summary}

A set of experimental tests were performed on the prototype. Result showed that static and rotational displacements are $15 \mu \mathrm{m}$ and $0.2^{\circ}$, respectively. The rise time is $14.5 \mathrm{~ms}$, while the fall time is $3625 \mathrm{~ms}$ ( 3.6 seconds). The estimated resonance frequency from time response data is approximately $120 \mathrm{~Hz}$. Further investigation is required to analyze the exact reason for the large value of the fall time. The chip circuit was integrated with a high resistance $(1 \mathrm{M} \Omega)$ to preventing short-circuit. 


\section{Chapter 5}

\section{Conclusions and Recommendations}

The following major objectives have been achieved in this thesis:

- A double-layer (Poly1+Poly2) MEMS repulsive actuator was designed and developed using surface micromachining process, PolyMUMPs, to produce a large out-of-plane force. The estimated out-of-plane force is $40 \mu \mathrm{N}$. The footprint area of the complete device is $10 \mathrm{~mm} \times 10 \mathrm{~mm}$.

- The stiffness of the platform was increased using supported beams, double thickness actuator, and electrostatic springs to hold a mass of $1 \mathrm{mg}$. All these options are implemented under the constraints of the PolyMUMPs process.

- A Faraday shield layer was designed and developed, compatible with the PolyMUMPS process, to avoid the unwanted electrostatic attractive effect in the double thickness MEMS actuator.

- The static and dynamics characteristics of the prototype were measured. The prototype is able to lift and rotate a platform of size $2.4 \mathrm{~mm}$ to $15 \mu \mathrm{m}$ and $0.3^{\circ}$ from its initial position at $120 \mathrm{~V}$. The average settling time in the rise and fall directions are found to be $14.5 \mathrm{~ms}$ and $36425 \mathrm{~ms}$ (3.6 seconds), respectively, with no significant overshoot in either direction. The resonance frequency was computed through the time response to be approximately $120 \mathrm{~Hz}$. The expected out-of-plane force is $40 \mu \mathrm{N}$ at $100 \mathrm{~V}$. 


\subsection{Limitations of Current Design}

1. The payload platform was connected to the actuators through a rigid beam to increase the stiffness of the platform. Due to rigid connections, the rotational motion of the platform about the desired axis could not be obtained. A stroke introduced by one actuator is transferred to the other actuators through the platform and a combined motion (translation + rotation) is observed in the xy plane, instead of only an x-axis or y-axis rotation.

2. In the prototype, the performance of the shielded layer is limited at high voltage. As a result, the moving fingers touch the connected beam of unaligned fixed fingers, leading to a short-circuit incident.

3. The residual stress effect of the double-layer PolyMUMPs structure was not considered during the simulation. Therefore, noticeable differences are observed in the static measurements in comparison to the simulation results.

\subsection{Recommendations to Improve the Design}

1. It is recommended that in a future design, the platform be connected with stiffer springs rather than the rigid beam to produce the desired rotations about the $\mathrm{x}$-axis and $\mathrm{y}$-axis. These springs will also absorb the releasing stress, preventing, additionally, an initial elevation of the platform.

2. Since the connected beam of the unaligned finger is not enclosed completely, the current shield layer is not very effective. The shield layer can be used more effectively by grounding it rather than leaving it neutral [29]. Therefore, it is recommended that the shield layer be grounded in any future design. 
3. In future design, residual stress effect should encounter in computational analysis of double-layer structure in order to predict the expected results.

\subsection{Future Work}

The out-of-plane force is estimated based on simulation result. The characteristics of the prototype are not examined under the load condition. Therefore, it is recommended that a load test be conducted in the future. The procedure to perform the load test is explained in subsection 4.4.1. The fall time response (3.6 seconds) is very large in comparison to the rise time $(14.5 \mathrm{~ms})$ response. Further investigation will be required to find the reasons.

If the prototype fabrication is not restricted to the commercial PolyMUMPs process, then other designs can also be produced for large out-of-plane force. In Appendix C, a double electrode MEMS repulsive actuator is discussed. The output force of a double electrode single actuator is $18 \mu \mathrm{N}$ at $100 \mathrm{~V}$, which is nearly double the force of the single layer electrode. 


\section{REFERENCES}

[1] R. A. Syns, H. Zou and J. Stagg, "Micro-opto-electro-mechanical systems alignment stages with vernier latch mechanisms," Journal of Optics A: Pure and Applied Optics, vol. 8, pp. 305-312, 2006.

[2] D.Herman and M.Deeds, "Out-of-plane deflection MEMS actuator for projectile control surfaces". US Patent 8405012 B1, 26 March 2013.

[3] R. J. Calvet and R. Gutierrez, "MEMS digital linear actuator". US Patent 7583006 B2, 1 Sept 2009.

[4] B.Vikramaditya and N. B.J, "Microssembly of hybird mangetic MEMS," Journal of Micromechatronics, vol. 1, no. 2, pp. 99-116, 2001.

[5] D. O. Popa and H. E. Stephanou, "Micro and Meso Scale Robotoic Assembly," Center of Automation Technologies, Rensselaer Polytechic Institute, New York, USA, 2003.

[6] M. J. Sinclair, "A High Force Low Area MEMS Thermal Actuator," in ITHERM 2000: Proc. 7th Intresoc. Conf. on Thermal and Thermomechanical Phenomena in Electronic Systems, 2000.

[7] J. J. Yao and S. C. Arney, "Fabrication of High Frequecy Two -Dimensional Nanoactuators for Scanned Prob Devices," Journa. Microelectromech. Systems, vol. 1, pp. 14-22, 1992.

[8] H. Guckel and K. Fischer, "Closed loop controlled, large throw, magnetic linear microactuator with 1000 micrometers structure height," in Proc. 11th Int. Workshop on MicroElectro Mechanical Systems pp 414-418, Heidelberg, 1998.

[9] V. P. Jaecklin and C. Linder, "Micromechanical Comb Actuators with Low Driving Voltage," Journal of Micromechanics. Microengineering., vol. 2, pp. 250-255, 1992.

[10] A. Selvakumar and K. Najafi, "Vertical Comb Array Microactuators," Journal Microelectromech. Syst., vol. 12, pp. 440-449, 2003.

[11] V. A. Henneken, M. Tichem and P. M. Sarro, "In-package MEMS-based thermal actuators for micro-assembly," Journal of Micromechanics and Microengineering, vol. 16, pp. 107-115, 2006.

[12] R. Gutierrez, "Autofocus mechanism for miniature cell phone cameras," Application Note - Tessera Inc., June 2011.

[13] J. Bareeau and P. Clark, "The optics of miniature digital camera modules," in Proc. of SPIE-OSA Conf. International Optics Design, SPIE Vol. 6342, 2006.

[14] C. Feng, "Auto-focusing lens with progressive variable focal element". US Patent 7149420 B1, 12 December 2006. 
[15] C. Gyoung and K. Young, "Optical system with optical image stabilization using a MEMS mirror". US Patent 2011/0181955 A1, 28 July 2011.

[16] C. Sheng and L. Dain, "Design and characterization of miniature auto-focusing voice coil motor actuator for cell phone camera applications," IEEE Transactions on Magnetics, vol. 45, no. 1, 2009.

[17] B.Berg, "Liquid lens technology-Principle of electrowetting based lenses and applications to imaging," in IEEE Conf. Publications, Micro Electro Mechanical Systems (MEMS), France, 2005.

[18] H. K. Lee, "Electroactive polymer actuator for lens-drive unit in autofocus compact camera module," Journal of Electronics and Telecommunications Research Institute, vol. 31, no. 12, pp. 695-702, 2009.

[19] M. K. Ojala, "Hydraulic optical focusing-stabilizer". US Patent 7627236 B2, 1 December 2009.

[20] C. Liu, "Electrostatic Sensing and Actuation," in Foundations of MEMS, New Jersey, Pearson/Prentice Hall, pp. 107-110, 2009.

[21] Y.-S. Kim, G. Nicholas and S. Gupta, "Design, fabrication and characterization of a single layer out-of-plane electrothermal actuator for a MEMS XYZ stage," in PerMIS '12 Proceeding of the Workshop on Performance Metric for Intelligent Systems, New York, 2012.

[22] T. E. Kenny, "Micromachined silicon tunnel sensor for motion detection," Applied Physics Letters, pp. 100-102, 1991.

[23] H. Cho and A. Cho, "A Bidirectional Magnetic Microactuator using Electroplated Permanent Magnet Arrays," Journal of Microelectromechanical Systems, no. 11, pp. 78-84, 2002.

[24] F. Tan and S. Alim, "Large-output-force out-of-plane MEMS actuator array," in Device and Process Technologies for MEMS, Microelectronics, and Photonics III, Australia, 2004.

[25] S. He and R. Ben Mrad, "Development of a High-Performance Microelectrostatic Repulsive-Force Rotation Actuator," Journal of Mircoelectromechanical Systems, vol. 19, no. 3, pp. 561-569, 2010.

[26] S. He and R. Ben Mrad, "Design, Modeling, and Demonstration of a MEMS Repulsive-Force OutOf-Plane Electrostatic Micro Actuator," Journal of Microelectromechanical Systems, vol. 17, no. 3, pp. 532-547, 2008.

[27] S. He, R. Ben Mrad and J. Chong, "Repulsive-force out-of-plane large stroke translation micro electrostatic actuator," Journal of Micromechanics and Microengineering, vol. 21, no. 7, June 2011.

[28] J.Chong, Siyuan He, Ridha Ben Mrad, "Development of Vector Display System Based on a Surface-Micromachined Micromirror," IEEE Transactions on Industrial Electronics, vol. 59, No.12, December 2012 
[29] Siyan He, R.Ben Mrad,'Development of a Novel Transltion Micromirror for Adaptive Optics," Proceedings of SPIE, vol. 5264, 2003.

[30] S.He, R.Ben Mrad, "Performance Assesment of a Multi-level Repulsive-force Out-of-plane Microelectrostatic Actuator,” Journal of Comput. Eng., vol. 31, No. 2, Spring 2006.

[31] S.He, S. Chang, "Experimental Verification of an Out-of-plane Repulsive-force Electrostatic Actuator Using a Macroscopic Mechanisim," Microsyst Technol (2009) 15:453-461, Springer.

[32] Da.Qiao, W.Yuan, "Design of an Electrostatic Repulsive-Force Based Vertical Micro Actuator," Proceedings of the $1^{\text {st }}$ IEEE International Conference on Nano/Micro Engineered and Molecular Systems, January 18-21, 2006, Zhuhai, China.

[33] F. Hu, W. Wang, "An Electrostatic MEMS Spring Actuator with Large Stroke and Out-of-plane Actuation," Journal of Micromech. Microeng., vol. 21, 2011

[34] J.Yao, F.Hu, “ Design and Analysis of Repulsive Electrostatic Driven MEMS Actuators,” Proceedings of SPIE (MEMS Adaptive Optics III), vol. 7209, 2009.

[35] J. Singh, A. Agarwal, “ A Novel Electrostatic Microactuator for Large Deflection in MEMS Applications," Journal of Thin Solid Films, 504 (2006), 64-68.

[36] K.Beng, Y. Cho, "Laterally Driven Electrostatic Repulsive -Force Microactuators Using Asymmetric Field Distribution," Journal of Microelectromechanical systems, vol. 10, No.1, March 2001.

[37] J. Carter and A. Crowen, PolyMUMPS Design Handbook, MEMSSCAP Inc., 2005.

[38] M. Faraday, "Experimental Researches in Electricity para 1174," 1849.

[39] I. Khan, J. Chong, R. Ben Mrad, S. He, and J. Schertzer, "Performance of an Electrostatic Actuated Micromirror in a Vacuum and Non-Vacuum Packaging," Proceedings of Industrial Electronics, Montreal, Oct. 2012.

[40] M. Hopcroft and W. Nix, "What is the Young's Modulus of Silicon," Journal of Micromechanical Systems, vol. 19, no. 2, 2010. 


\section{Appendix A}

\section{Prototype Design}

\section{A-1. 2 D Layout of Prototype Design in AutoCAD}

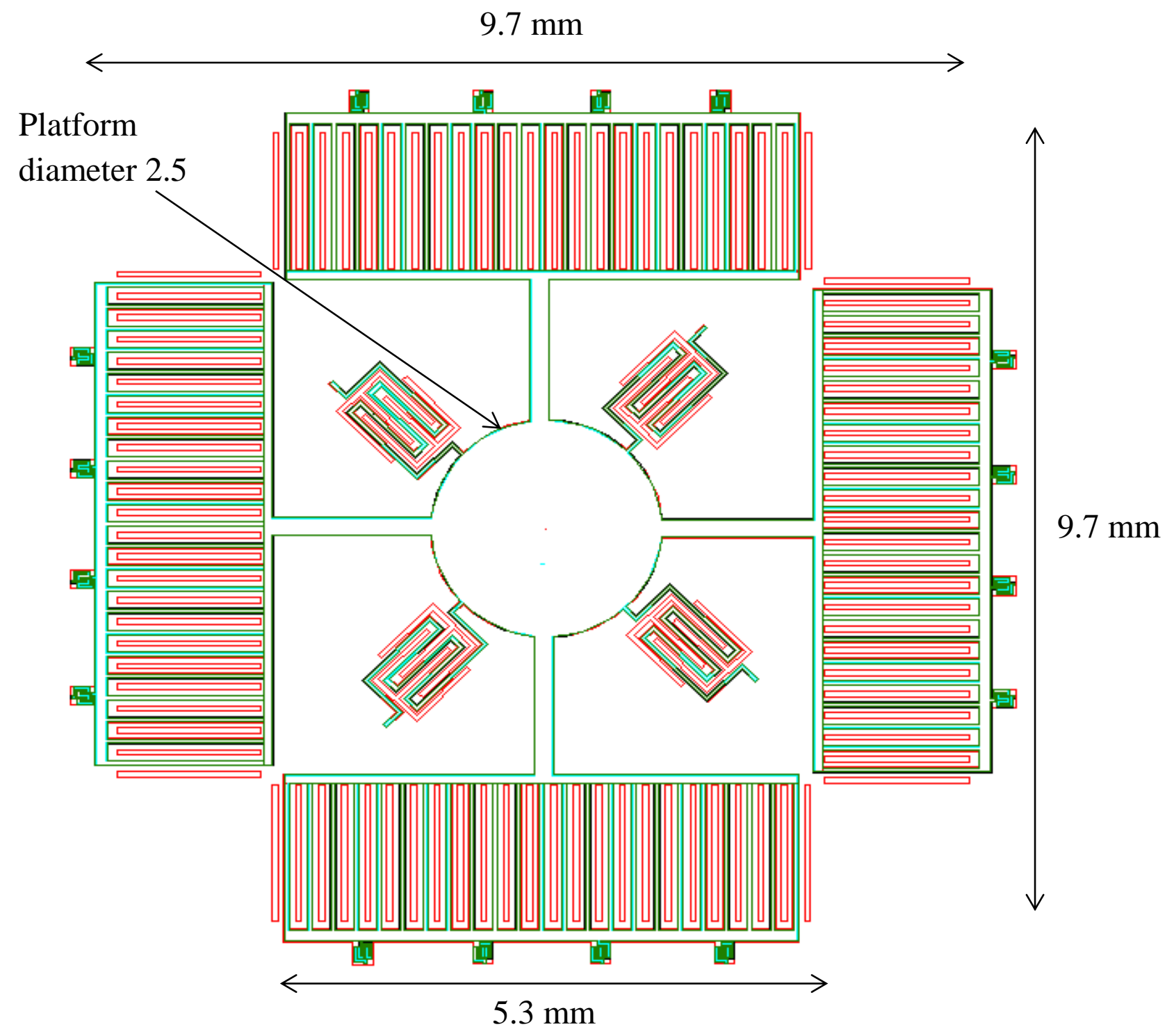

Figure A-1. AutoCAD mask layers Prototype. 
A-2. Bonding diagram of Prototype with CPGA84 package.

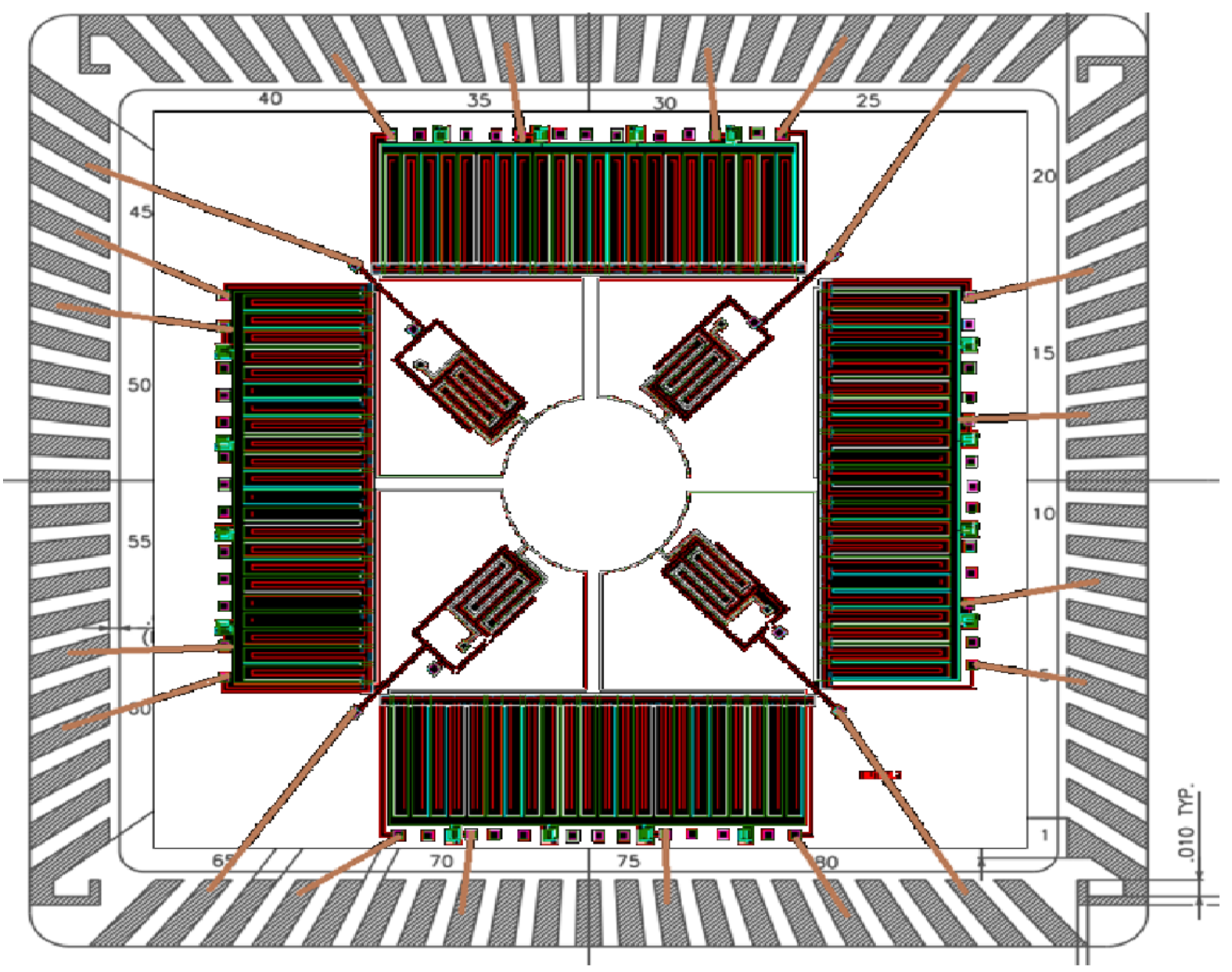

\begin{tabular}{|l|l|}
\hline Electrical Potentials & $\begin{array}{l}\text { Electrical Connections } \\
\text { Pin number for CPGA84 packaging }\end{array}$ \\
\hline Ground & $\mathbf{8 , 1 3 , 2 9 , 3 4 , 4 8 , 5 8 , 7 1 , 7 6}$ \\
\hline Voltage & $\mathbf{5 , 1 7 , 2 3 , 2 6 , 3 8 , 4 5 , 4 7 , 6 0 , 6 5 , 6 7 , 8 0 , 8 3}$ \\
\hline
\end{tabular}

Figure A-2. Bonding diagram for prototype packaging in CPGA 84. 


\section{Appendix B}

\section{Development Process of Prototype in PolyMUMPs}

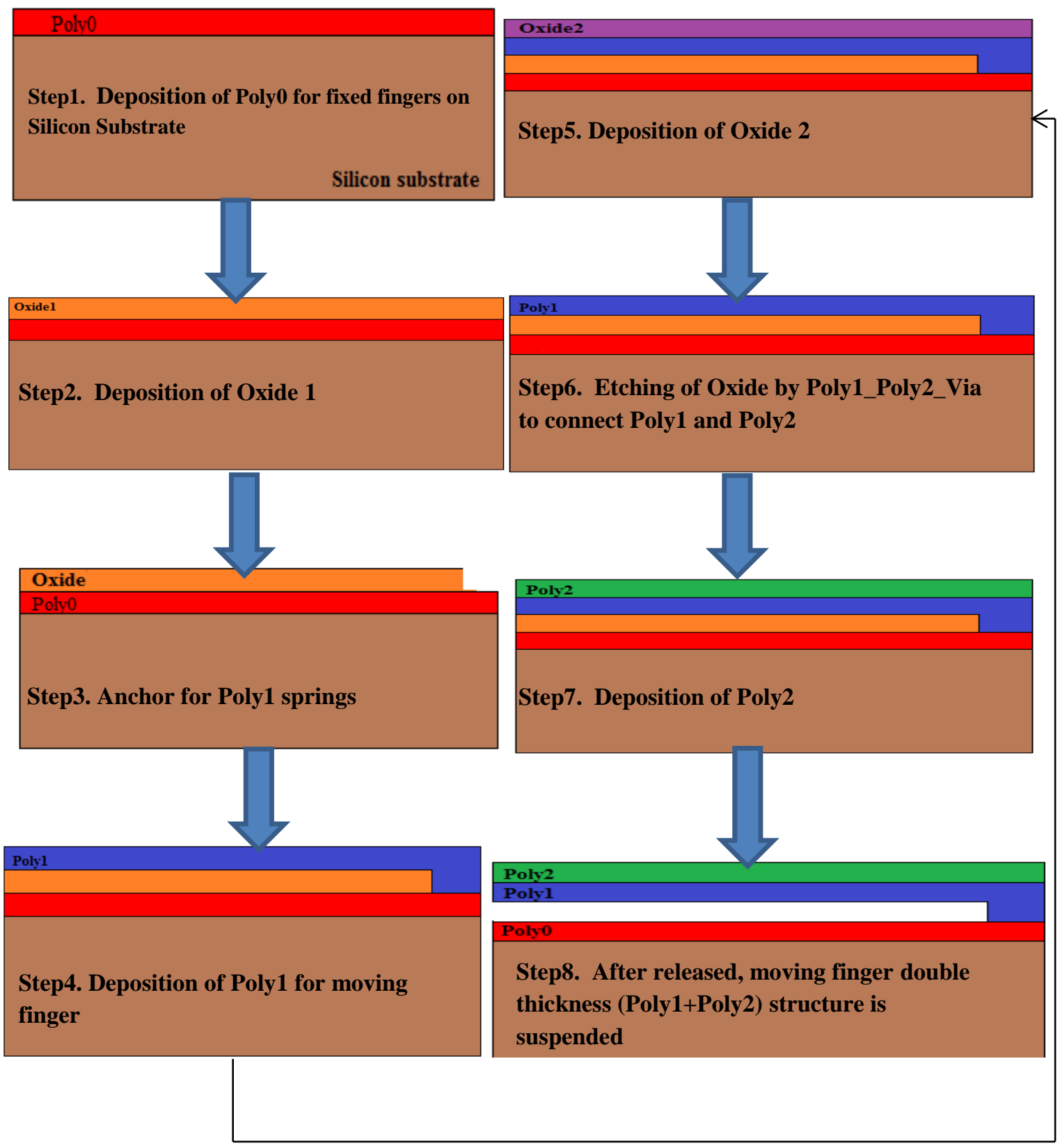

Figure B-1. Step by step PolyMUMPs process to develop double layer MEMS repulsive actuator. 


\section{$\underline{\text { Appendix C }}$}

\section{Double-Layer Electrode MEMS Repulsive Actuator}

\section{C.1 Double Layer Electrode MEMS Repulsive Actuator}

In order to increase the individual force of each actuator, a double layer electrode design option can also be considered if the fabrication process is not limited to PolyMUMPs. The unit length of the ground and electrode fingers is simulated using the Electrovolt software to determine the repulsive force between the fixed and moving fingers, where the finger width was set to $5 \mu \mathrm{m}$. The output force of double electrode layers is almost twice that of the single layer electrode as shown in Figure C-1.

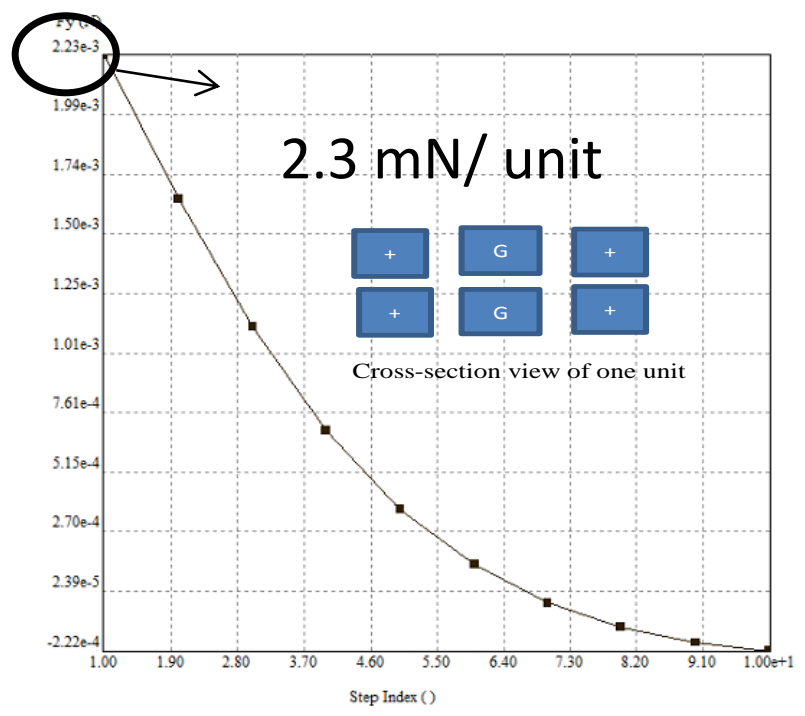

(a)

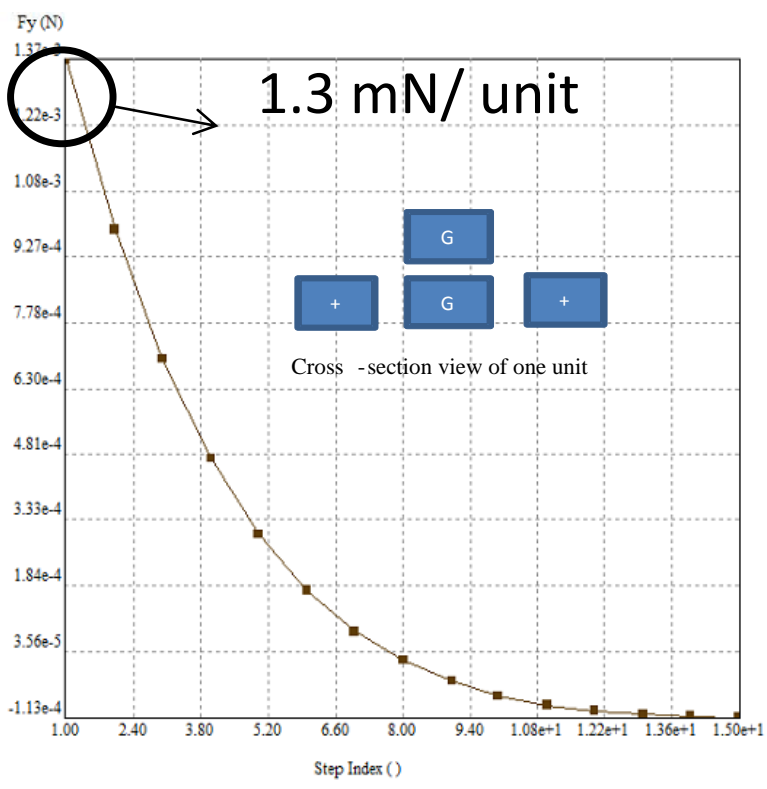

(b)

Figure C-1 Simulation result for output force in Electrovolt software (a) Double layer electrode (b) Single layer electrode. 
A 3D Coventor model of double layer electrodes and the simulation result of double layer electrode actuator are shown in Figure C-2. The output force of double layer electrode (18 $\mu \mathrm{N})$ is larger than that of single layer electrode but the design of double layer electrode is complicated.

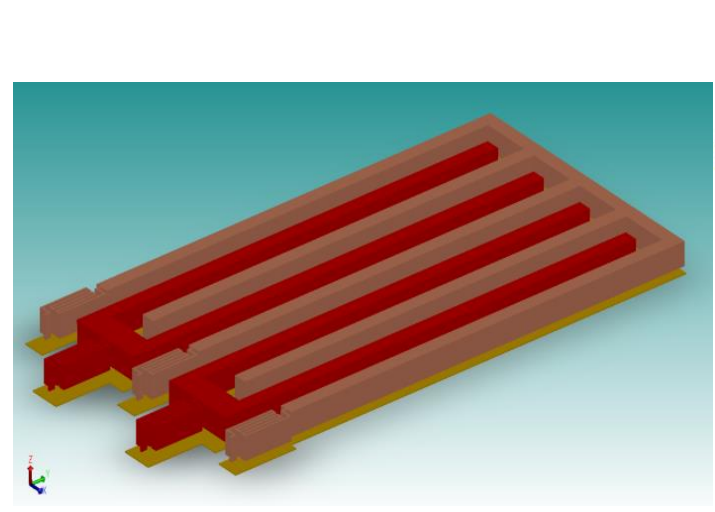

(a)

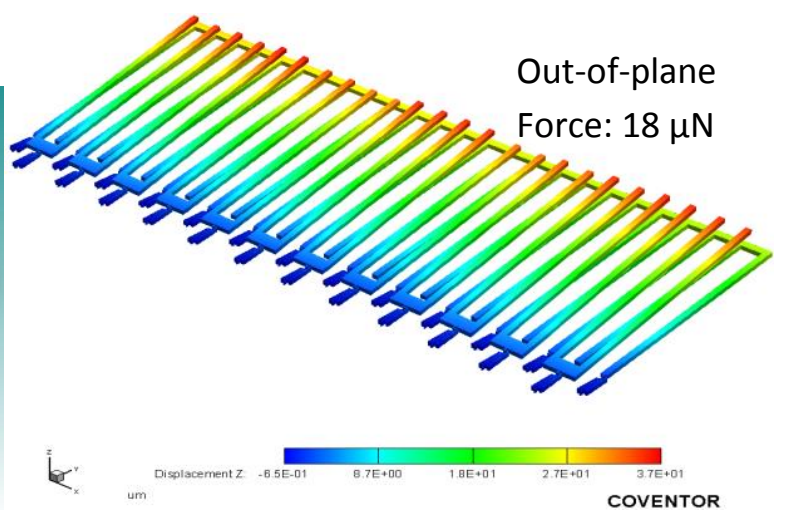

(b)

Figure C-2 Double-layer actuator model (a) 3D model of one unit double layer actuator (b) Simulation result of double electrode actuator at $100 \mathrm{~V}$. 